\title{
Supplementary information: The effect of complex optimal orbitals on atomization energies with the Perdew-Zunger self-interaction correction to density functional theory
}

\author{
Susi Lehtola
}

June 20, 2016

The scatter plots for the atomization energy errors for the 10 functionals studied in the present work are shown in Figures 1 - 36. In each figure, the division of the molecules into classes (A), (B), (C), and (D) has been illustrated with a vertical dashed black line. The plots also show vertical, solid blue lines illustrating the mean error $\mu$ in each class, as well as two vertical dashed, blue lines illustrating $\mu-\sigma$ and $\mu+\sigma$ where $\sigma$ is the root mean square deviation within the class. The $\mathrm{x}$ axes show the name of the molecule in the W4-11 database. 


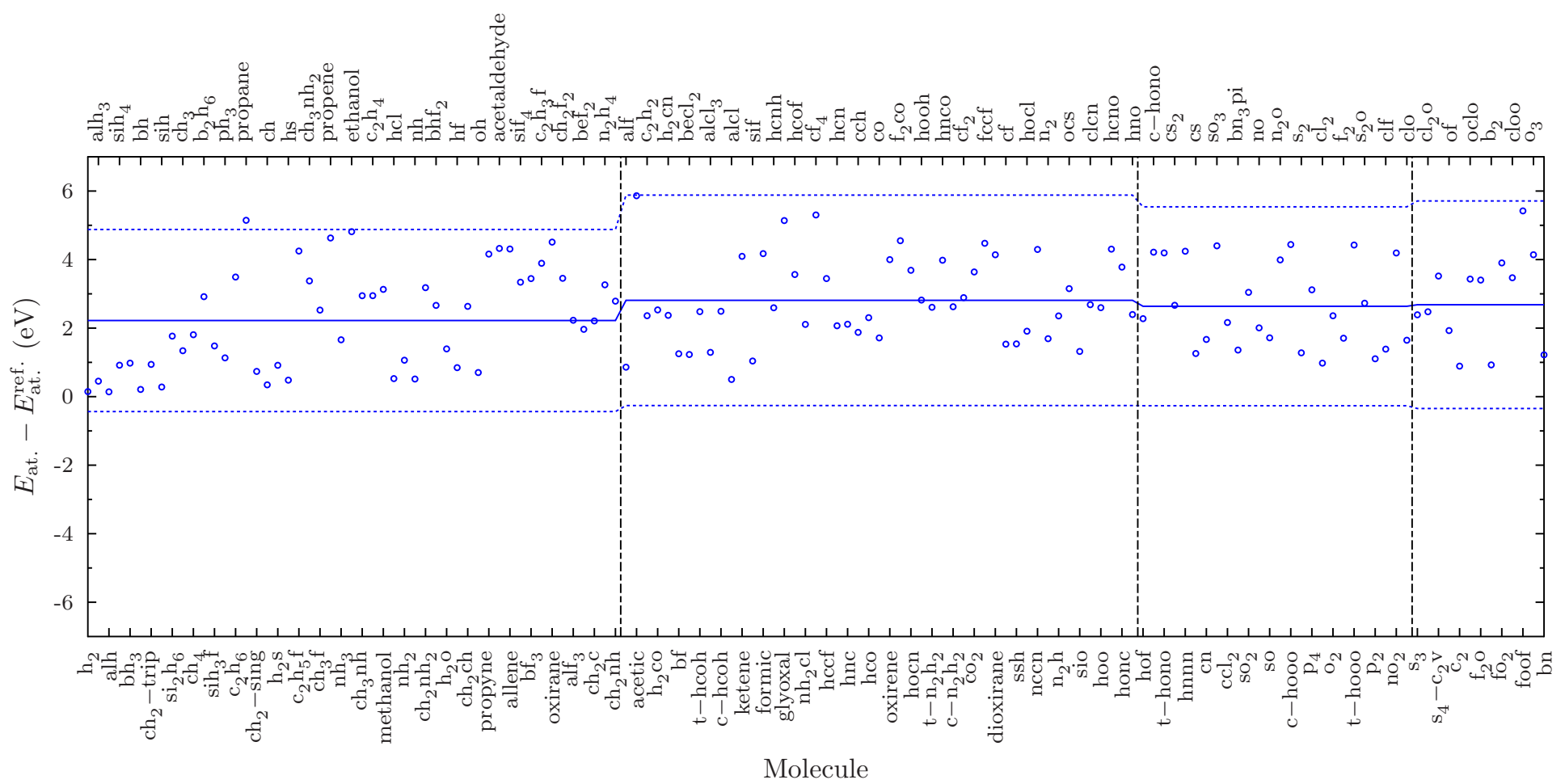

Figure 1: LDA KS-DFT results.

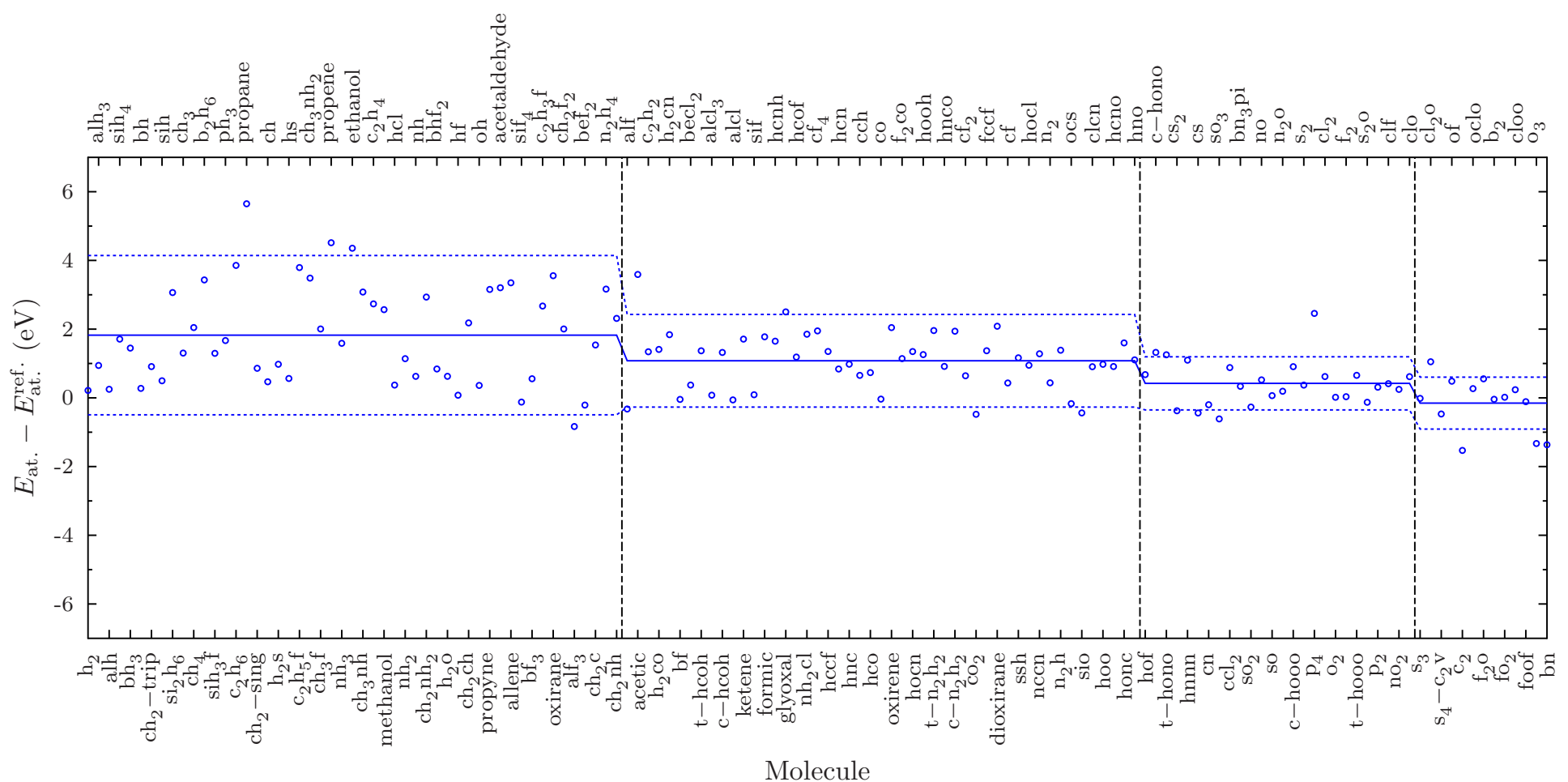

Figure 2: LDA PZ-RSIC results. 


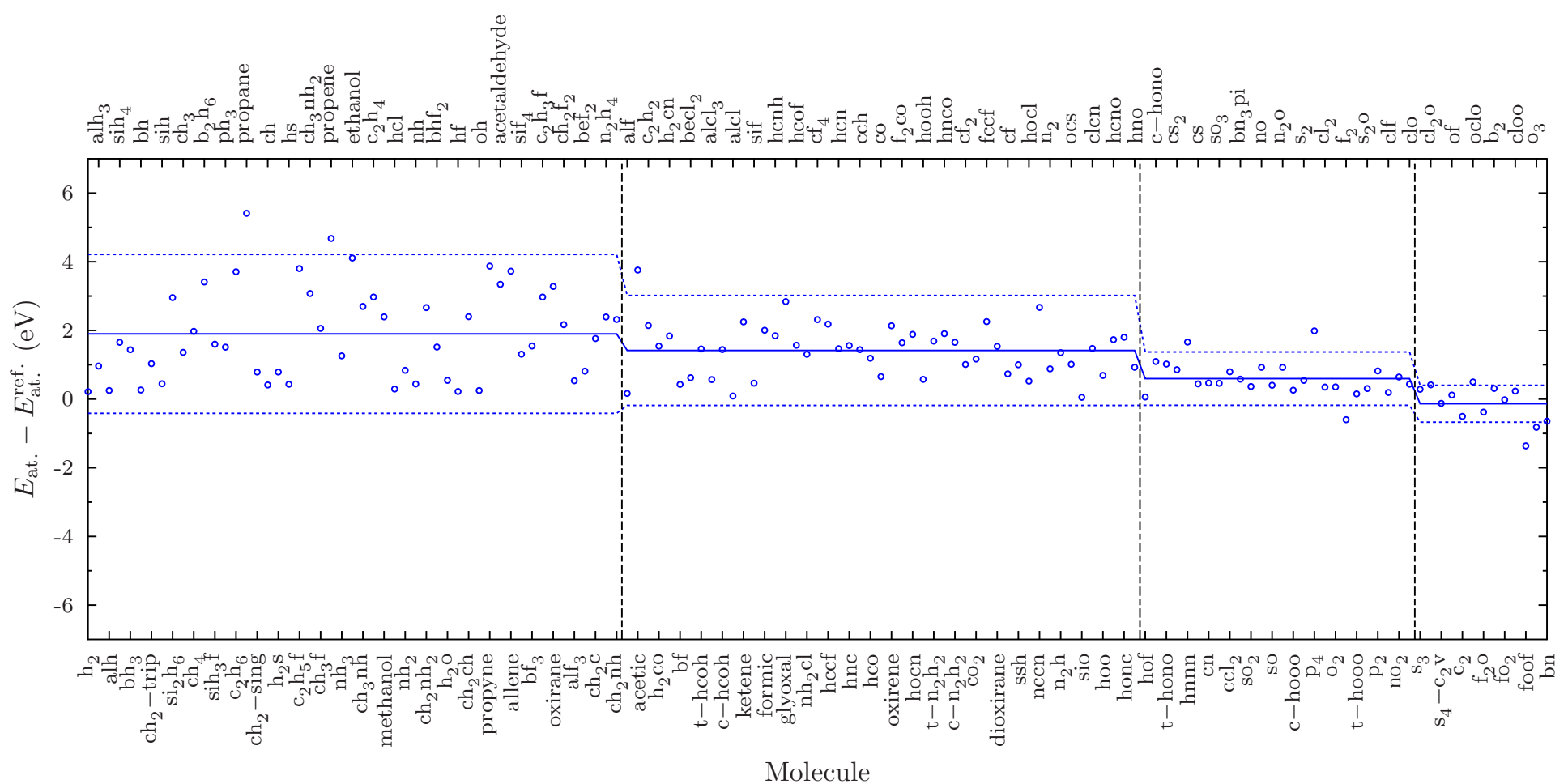

Figure 3: LDA PZ-SIC results.

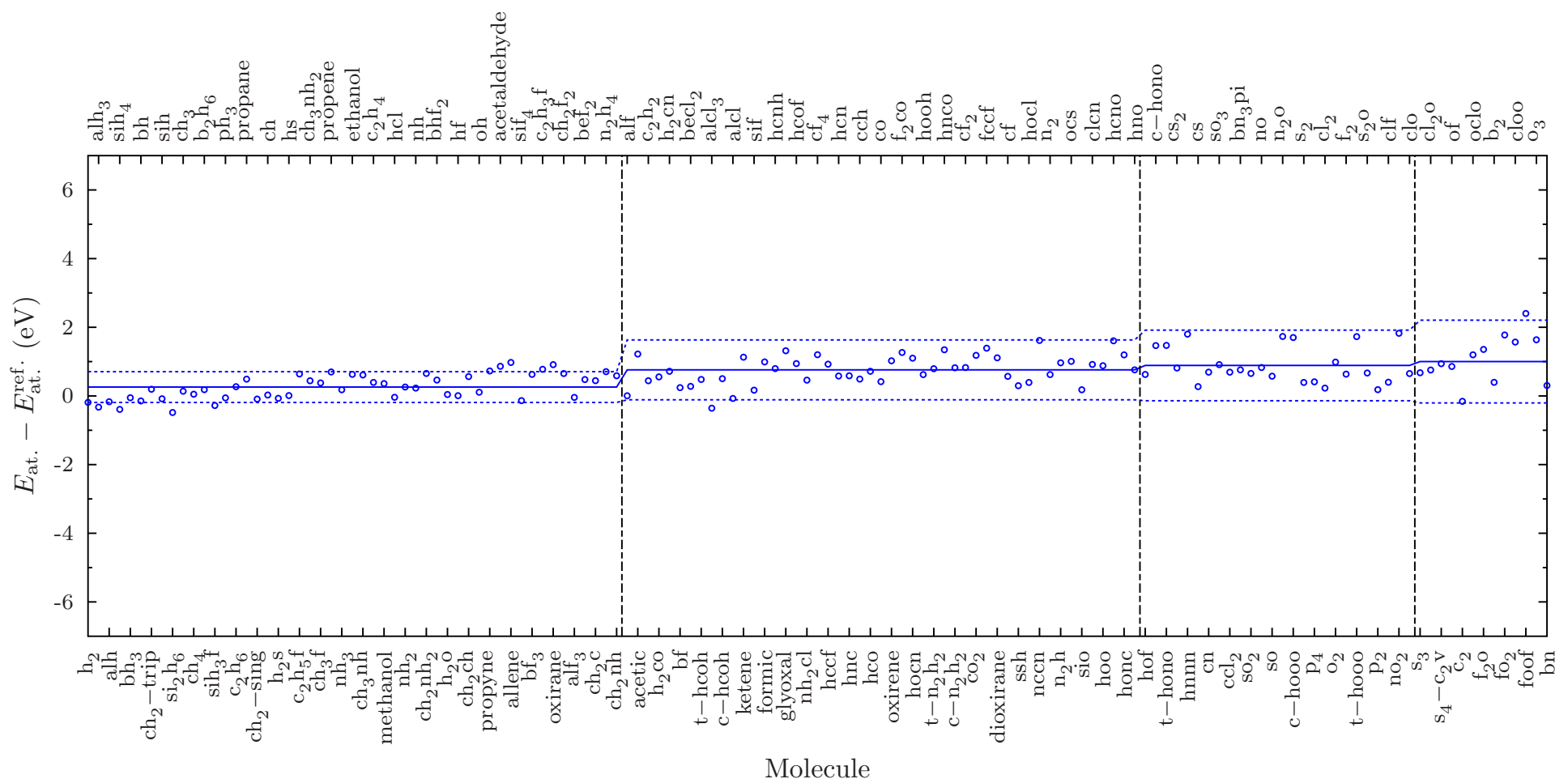

Figure 4: PW91 KS-DFT results. 


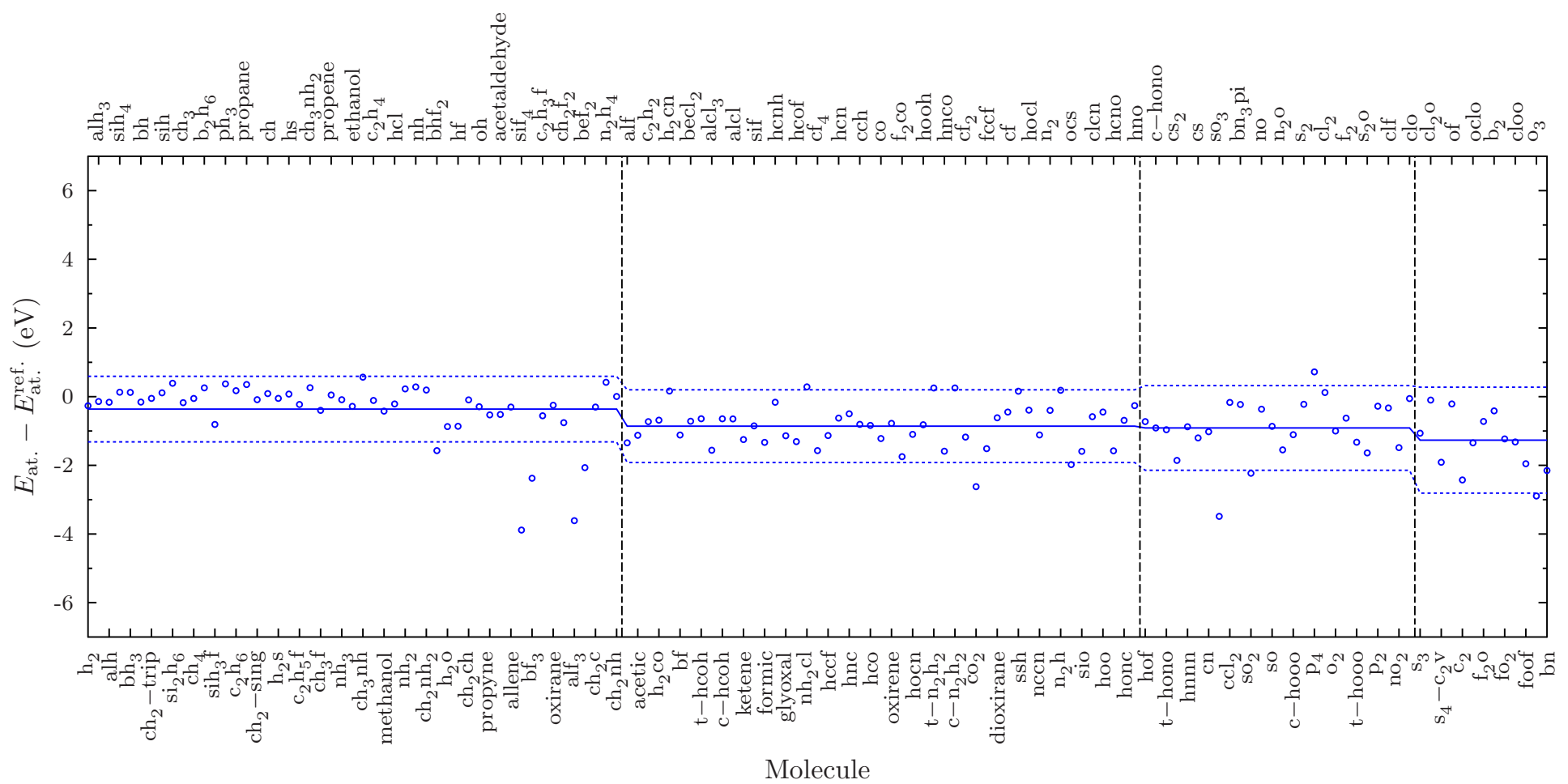

Figure 5: PW91 PZ-RSIC results.

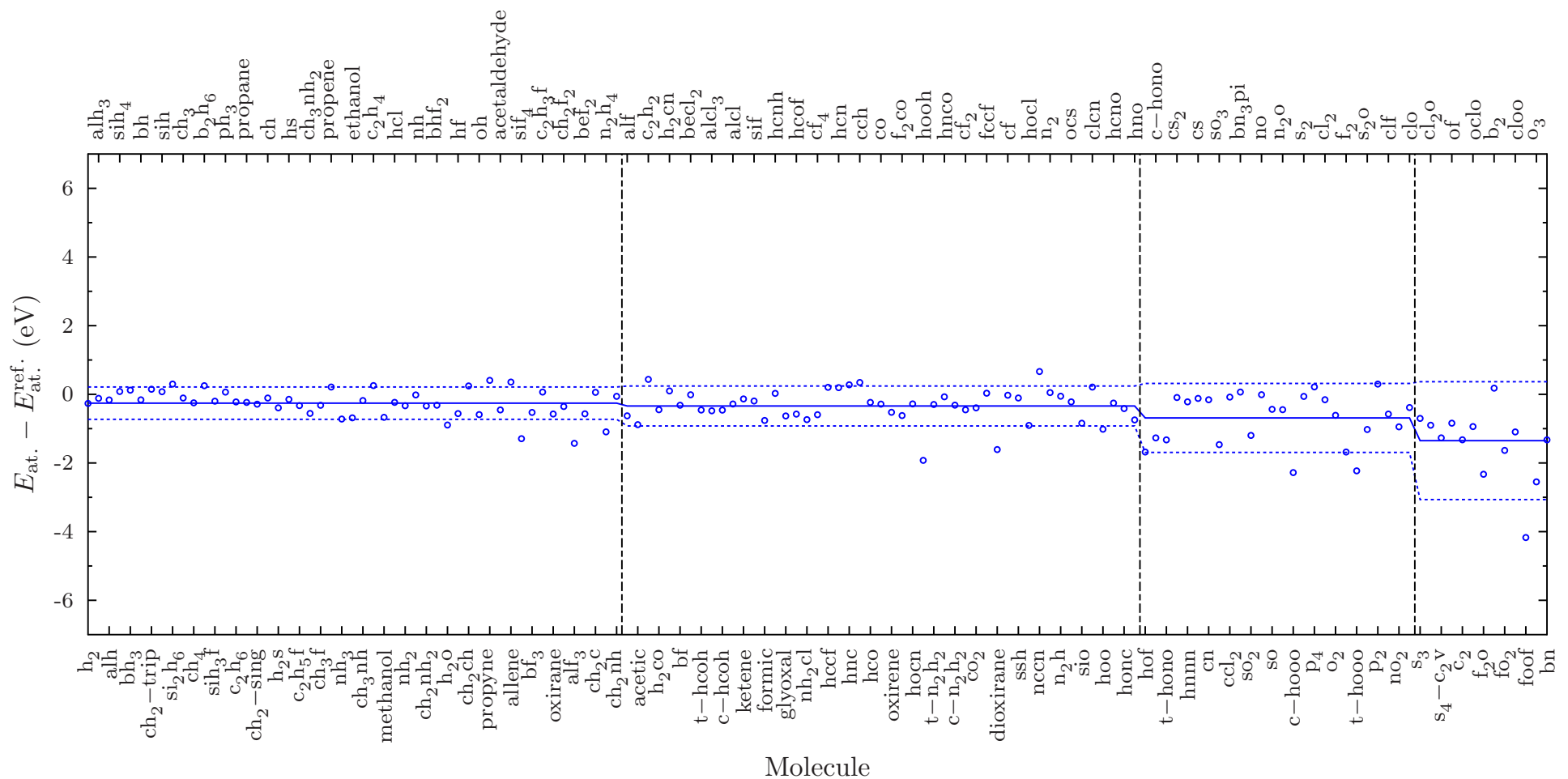

Figure 6: PW91 PZ-SIC results. 


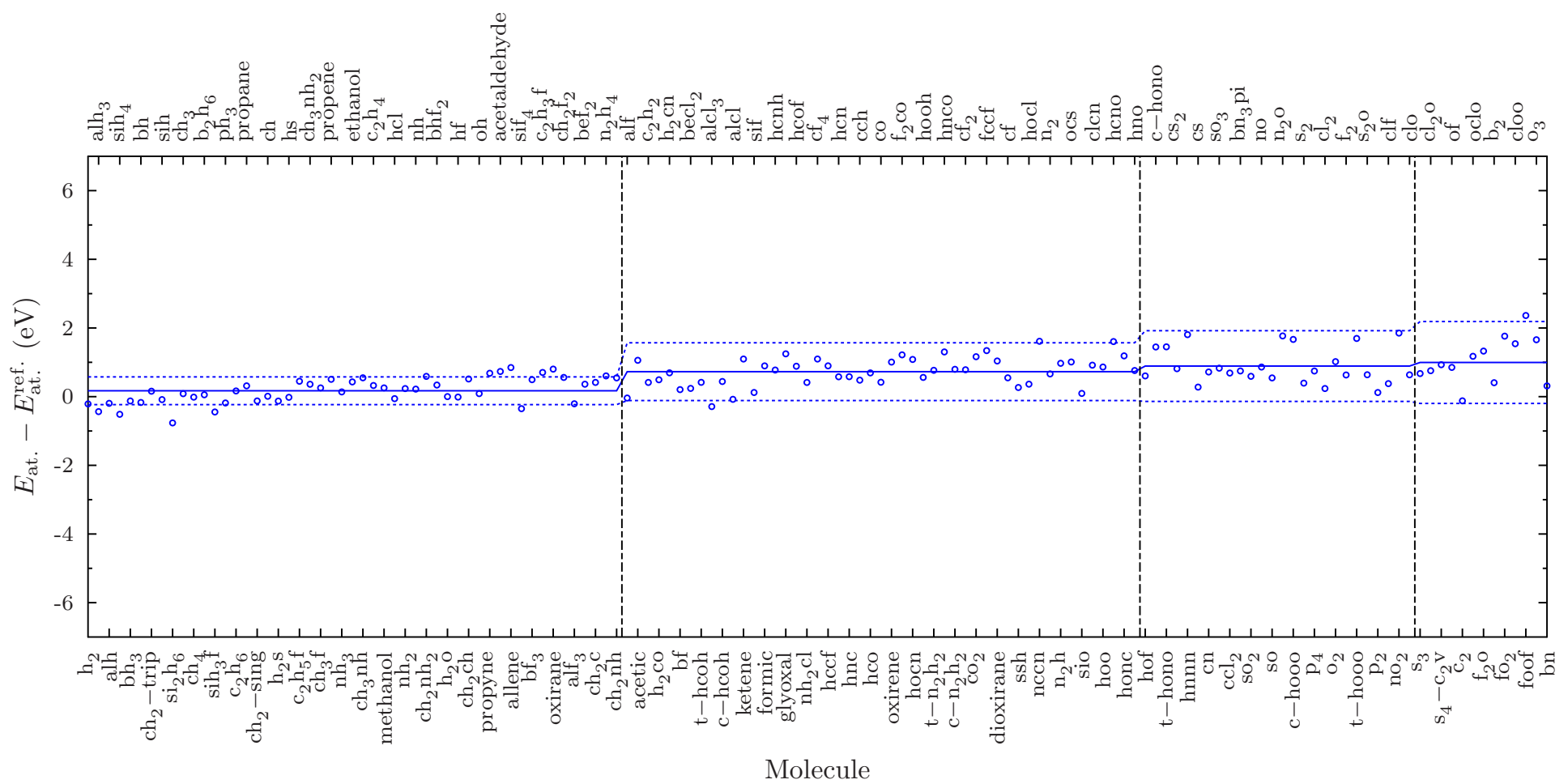

Figure 7: PBE KS-DFT results.

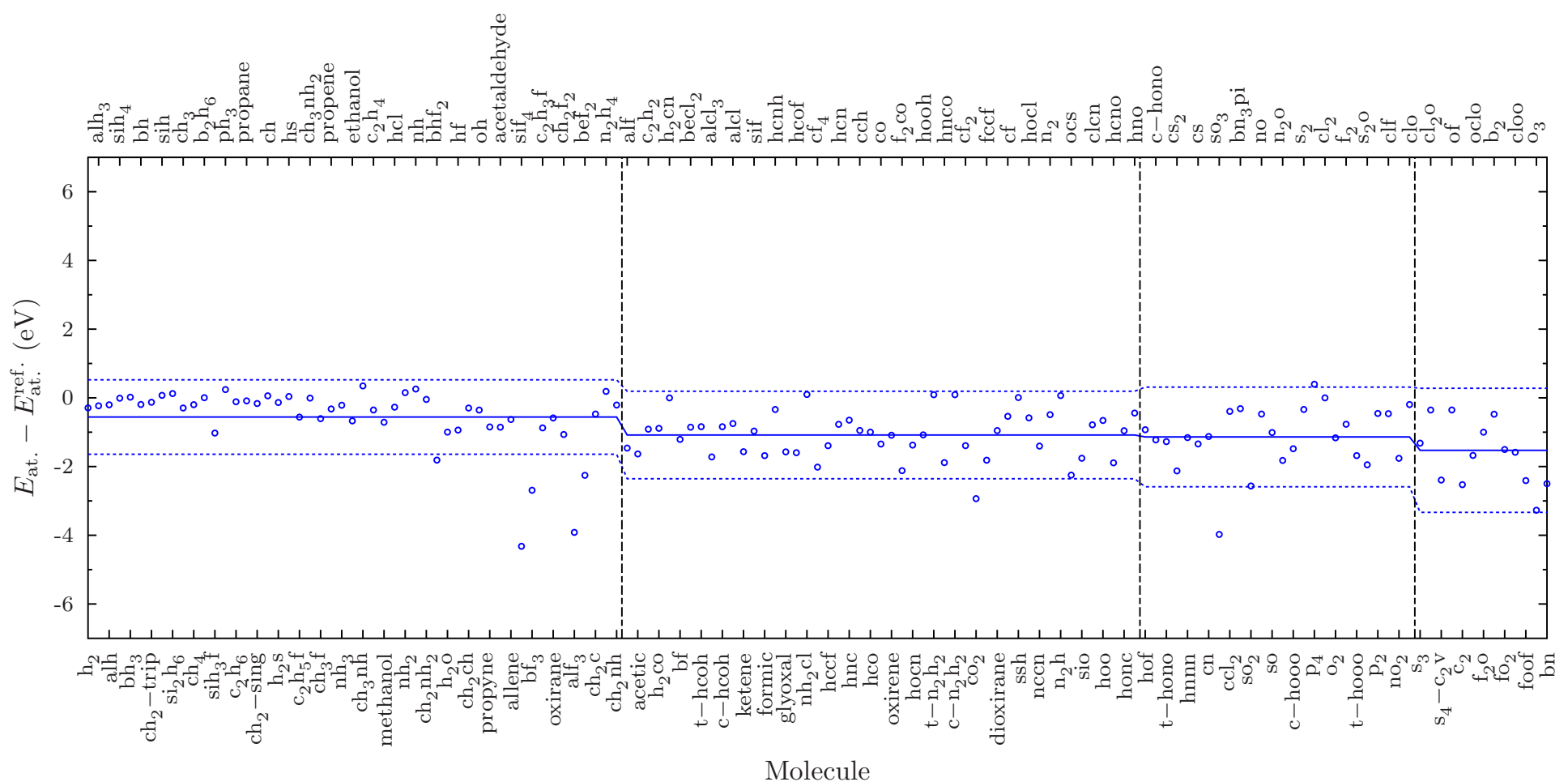

Figure 8: PBE PZ-RSIC results. 


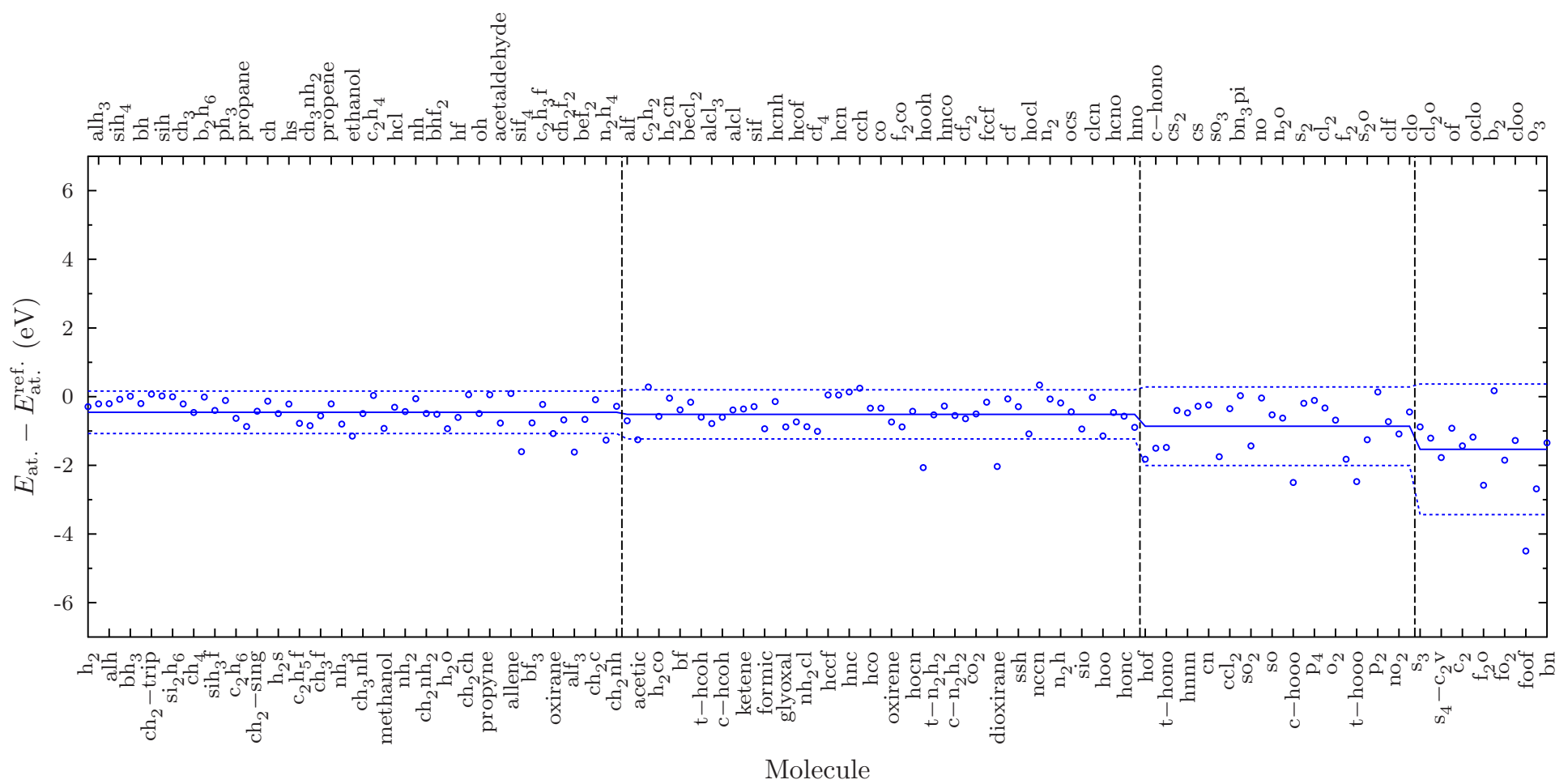

Figure 9: PBE PZ-SIC results.

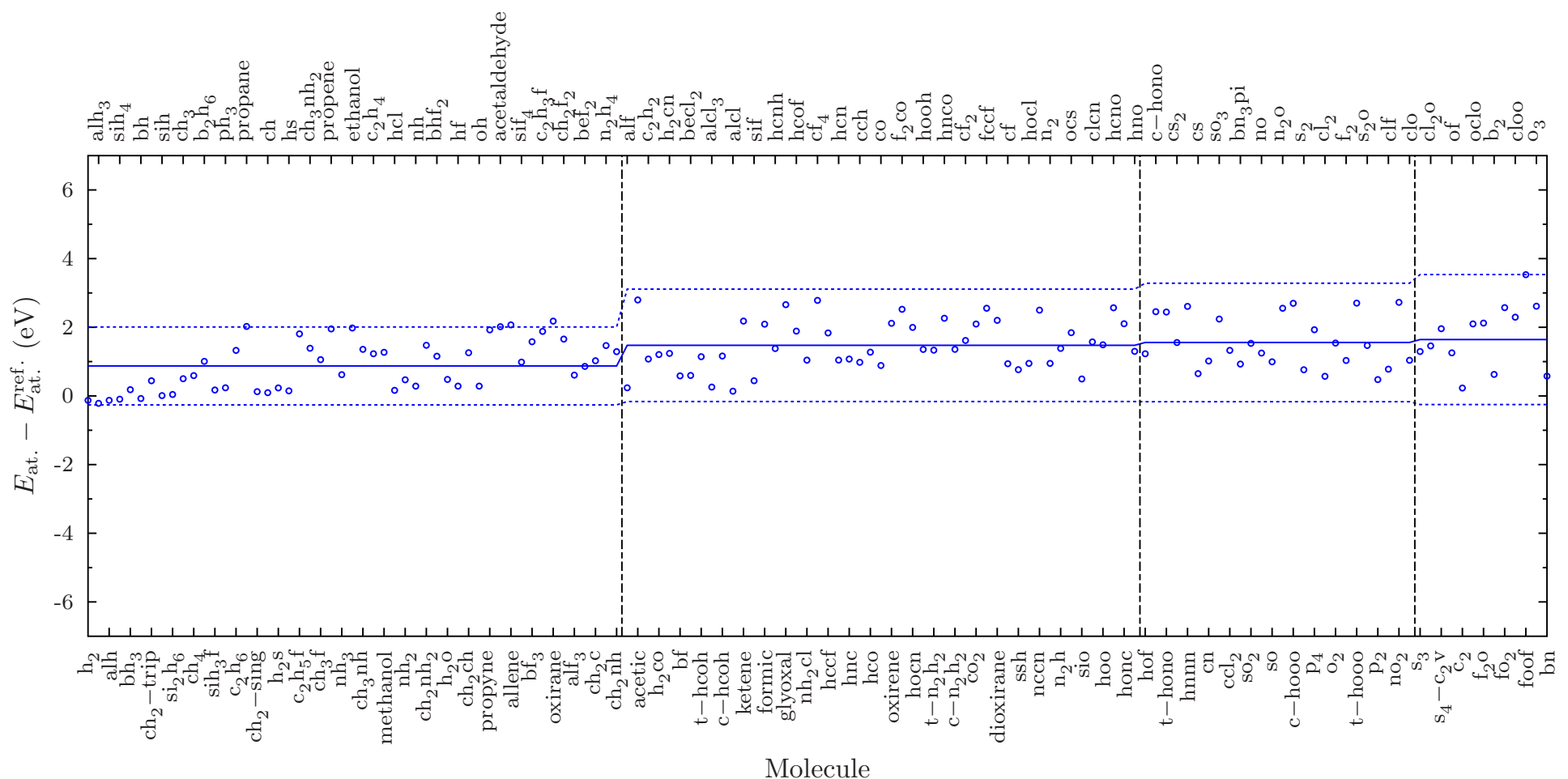

Figure 10: PBEsol KS-DFT results. 


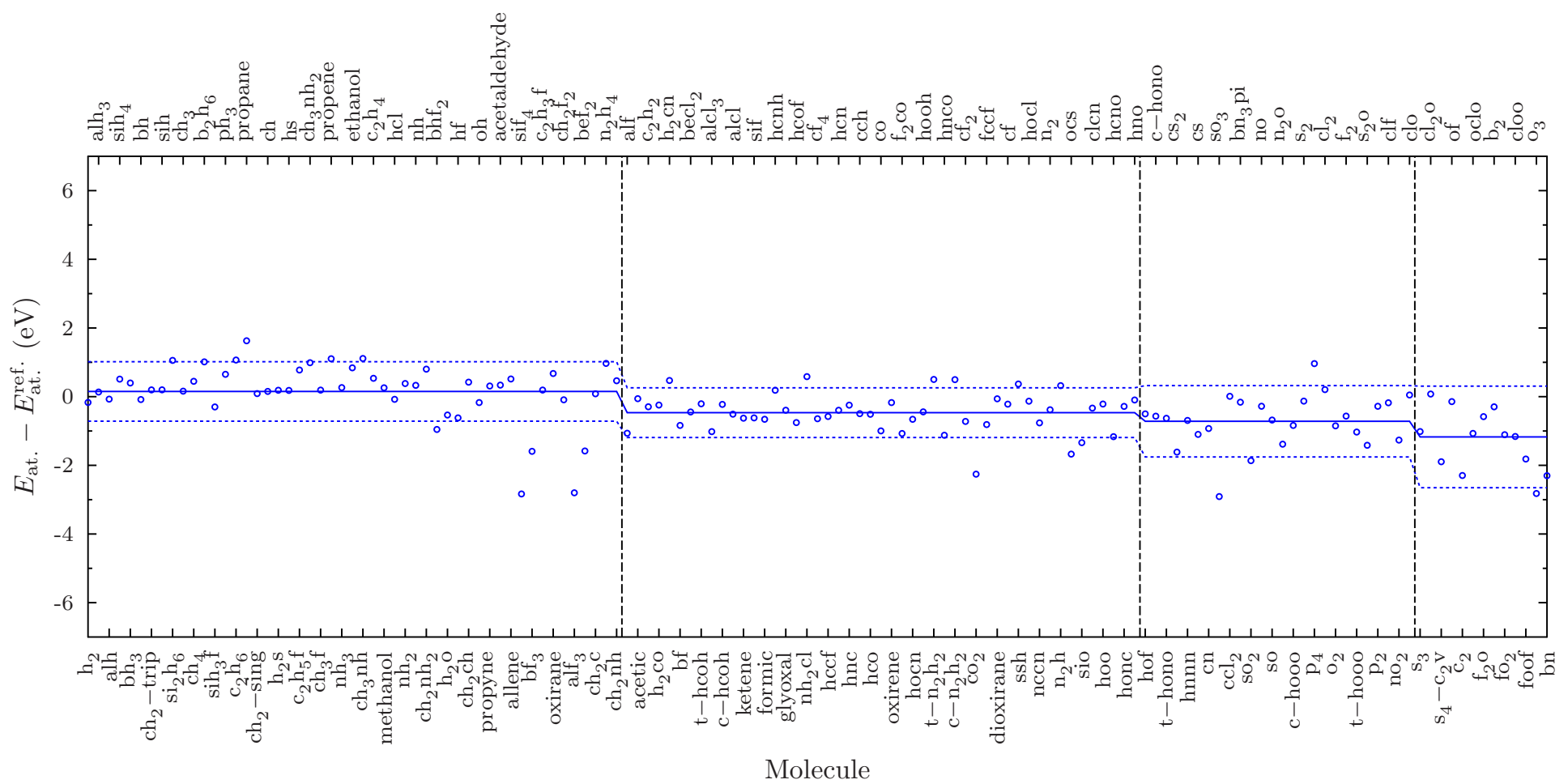

Figure 11: PBEsol PZ-RSIC results.

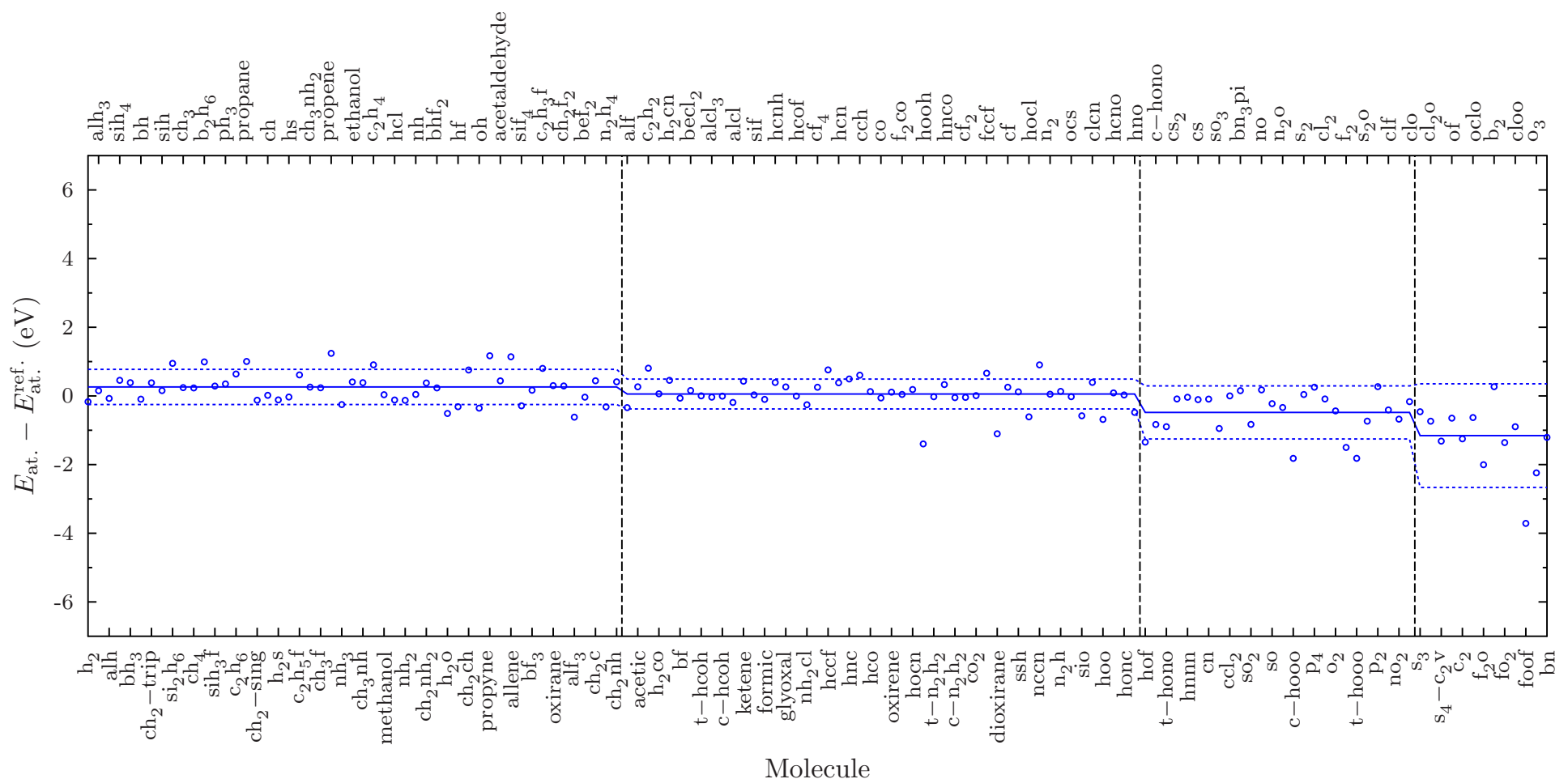

Figure 12: PBEsol PZ-SIC results. 


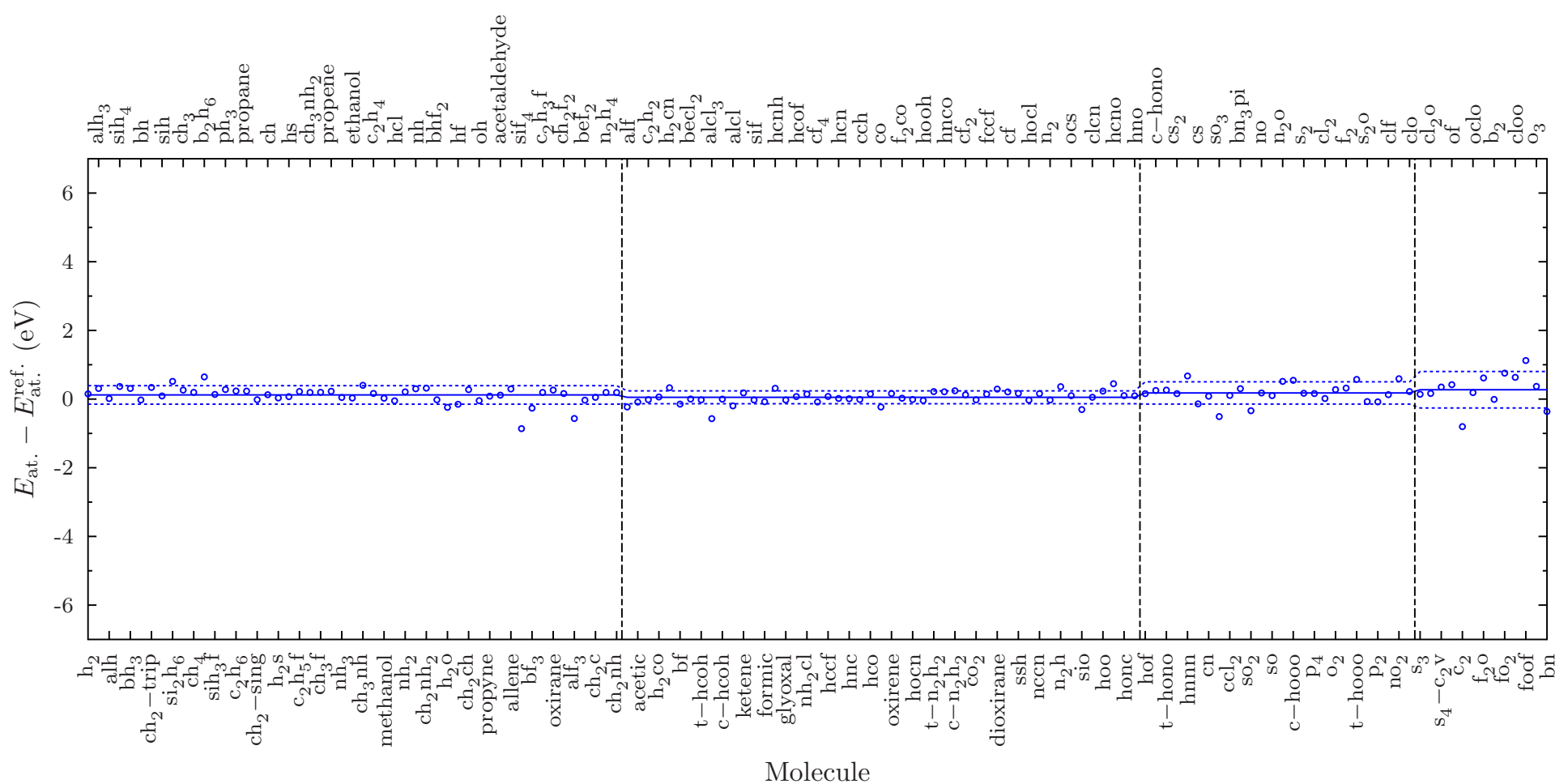

Figure 13: TPSS KS-DFT results.

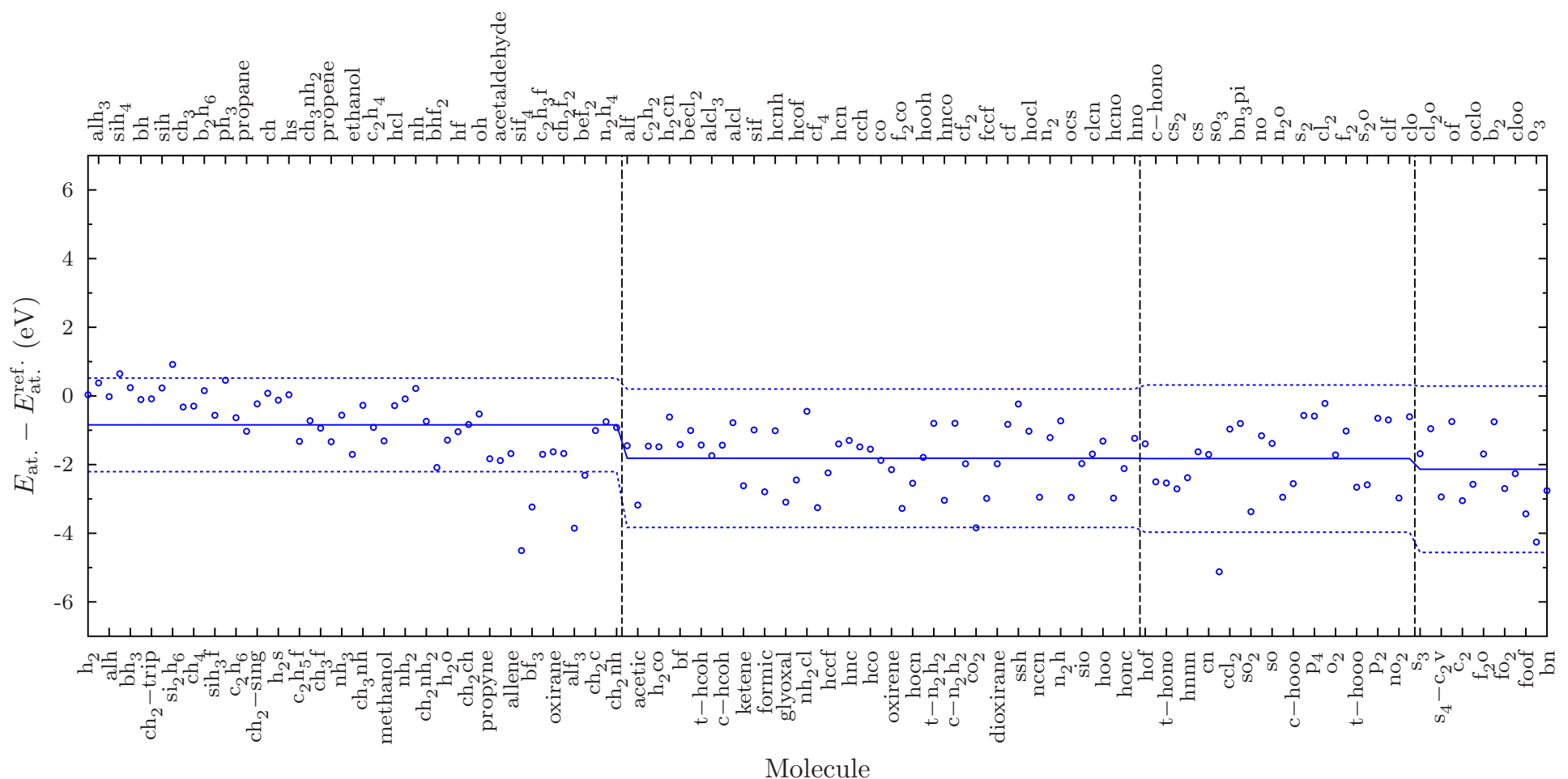

Figure 14: TPSS PZ-RSIC results. 


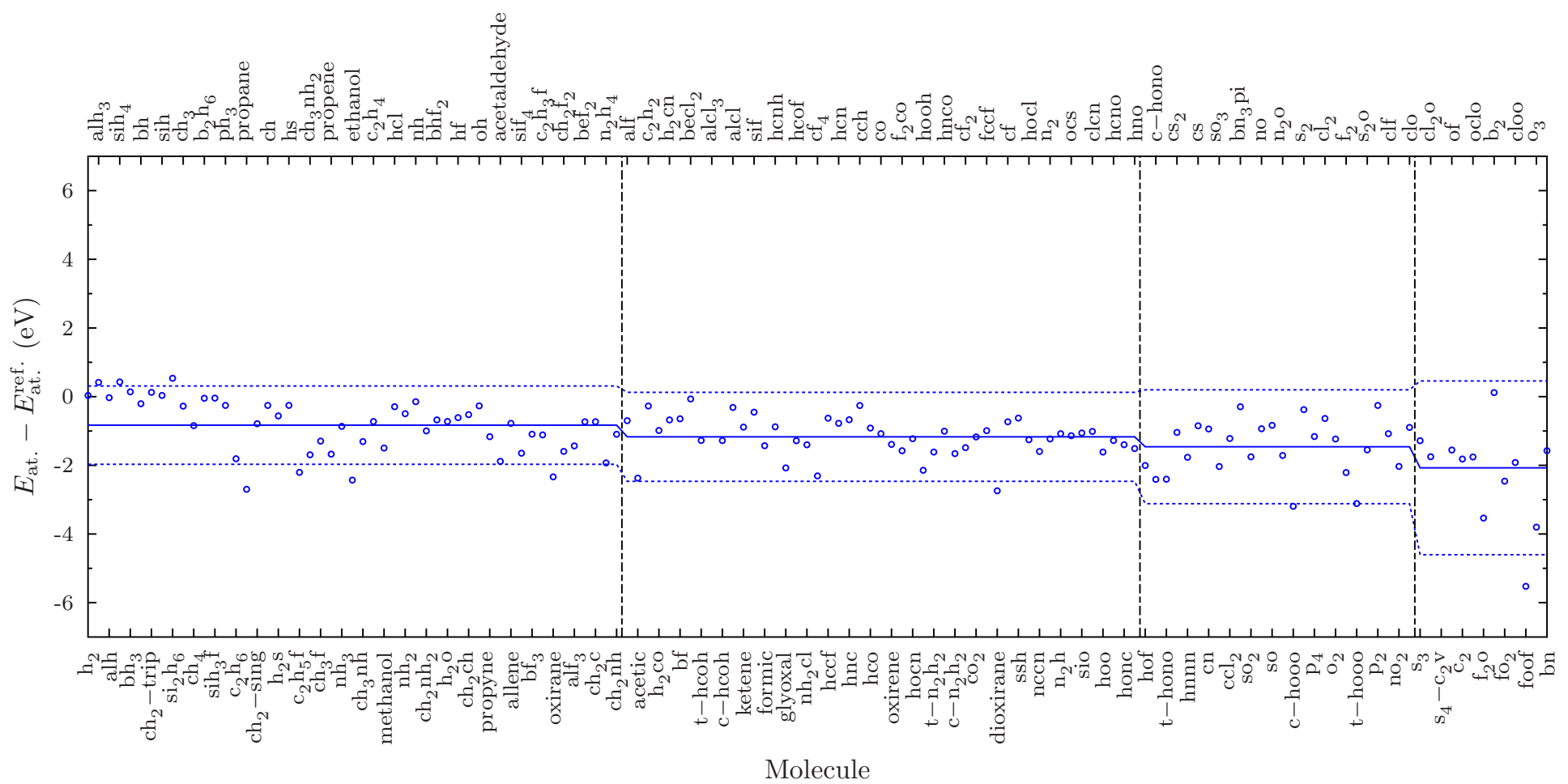

Figure 15: TPSS PZ-SIC results.

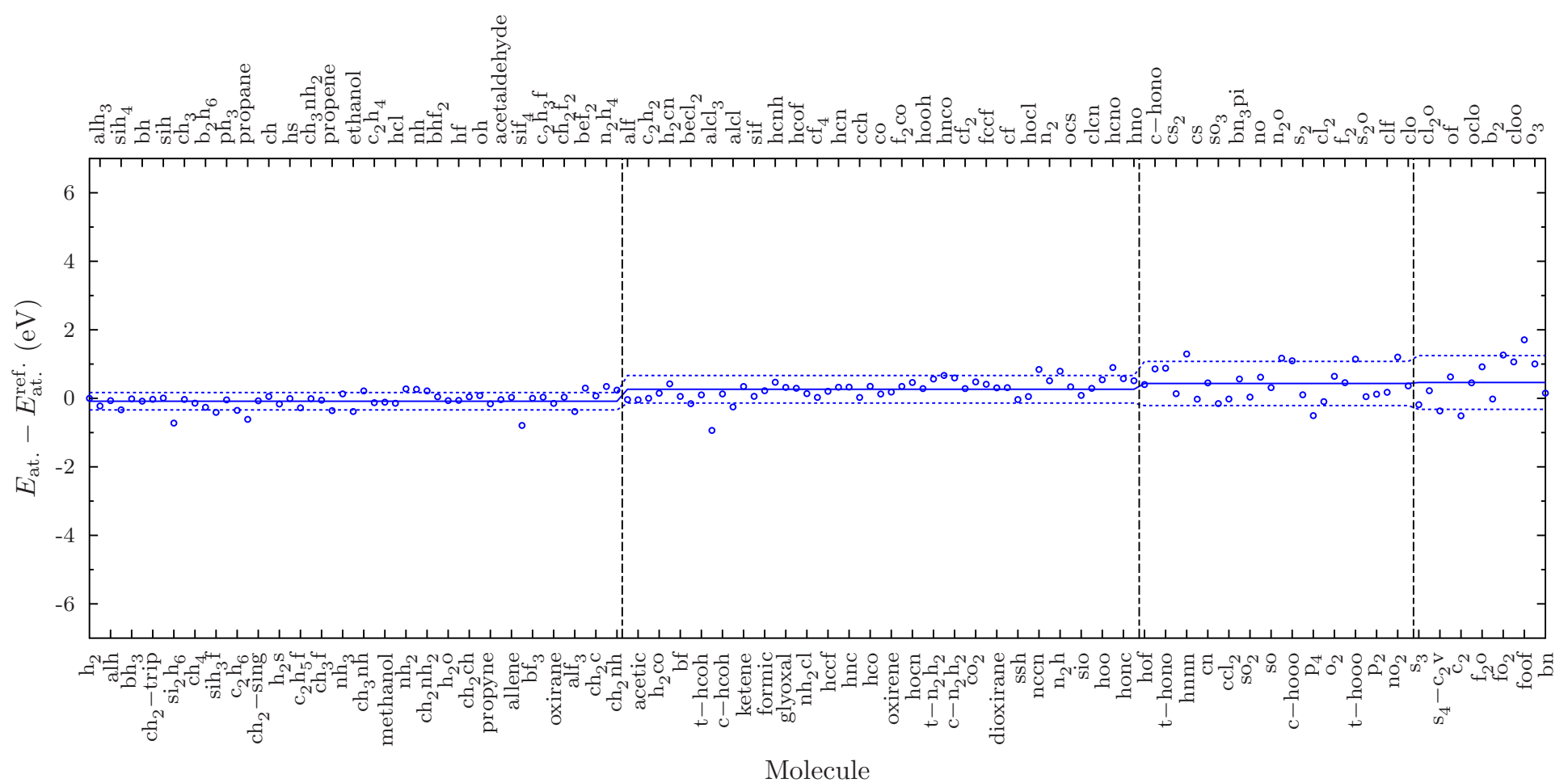

Figure 16: BLYP KS-DFT results. 


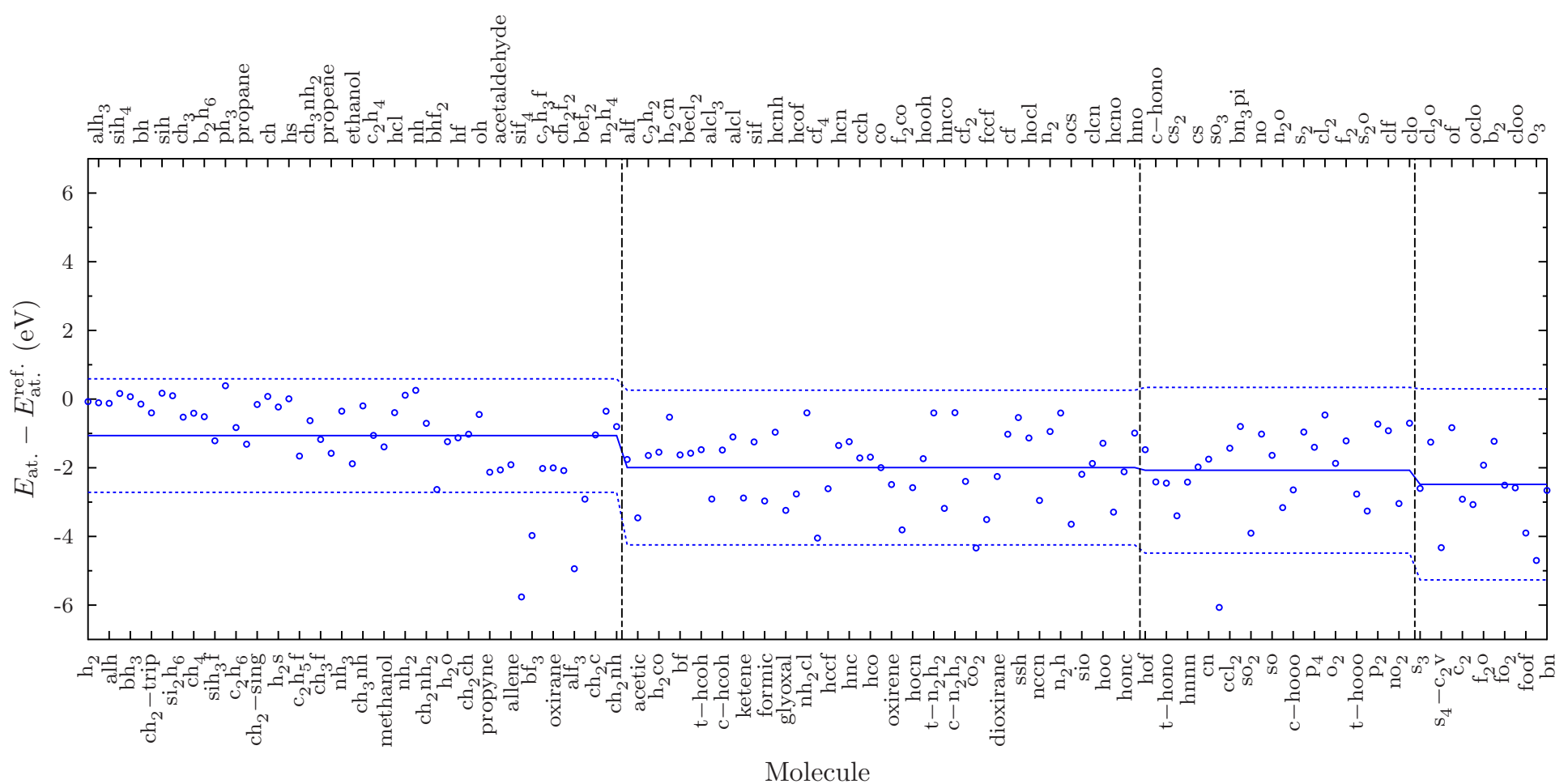

Figure 17: BLYP PZ-RSIC results.

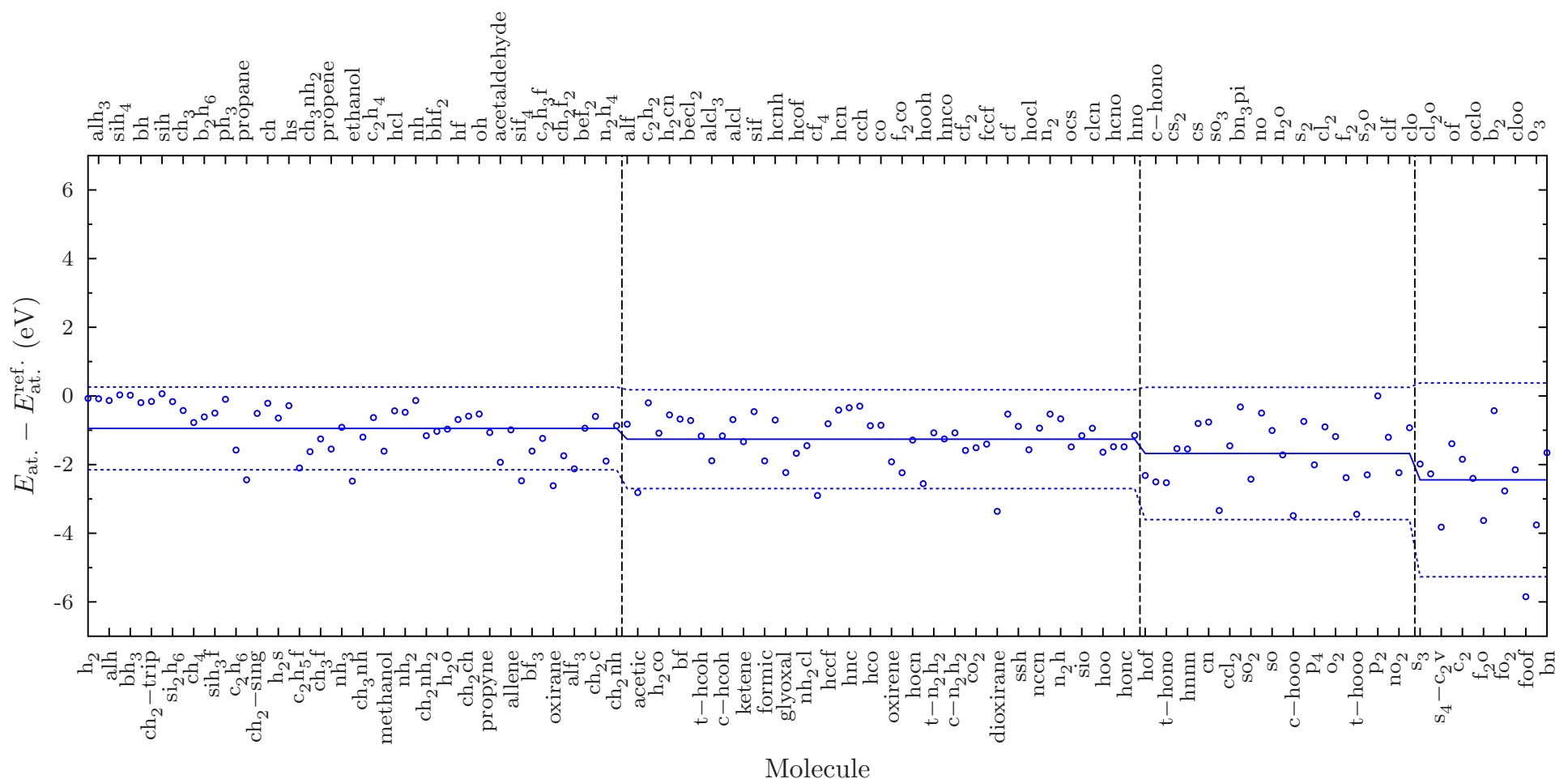

Figure 18: BLYP PZ-SIC results. 


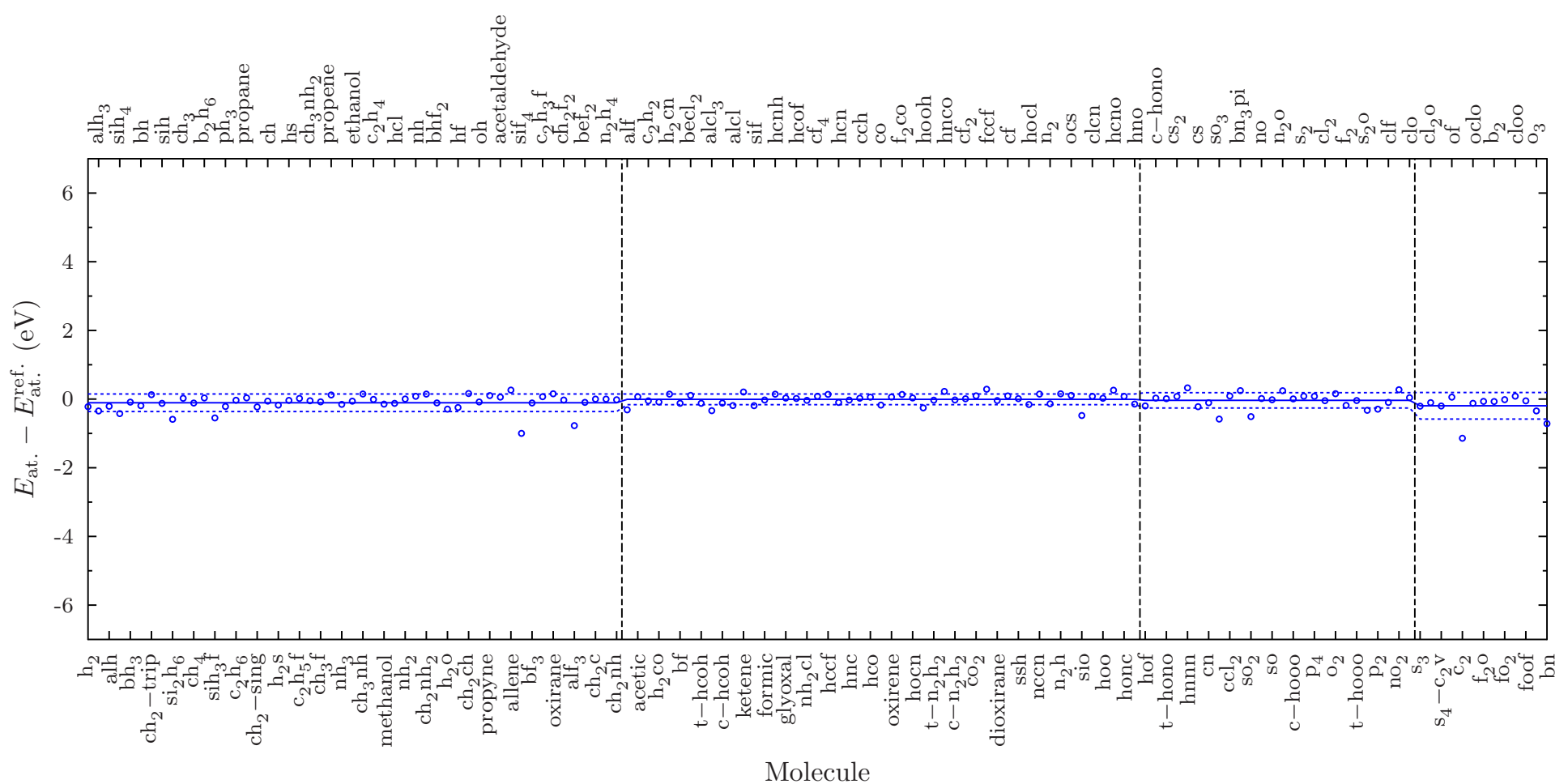

Figure 19: PBE0 KS-DFT results.

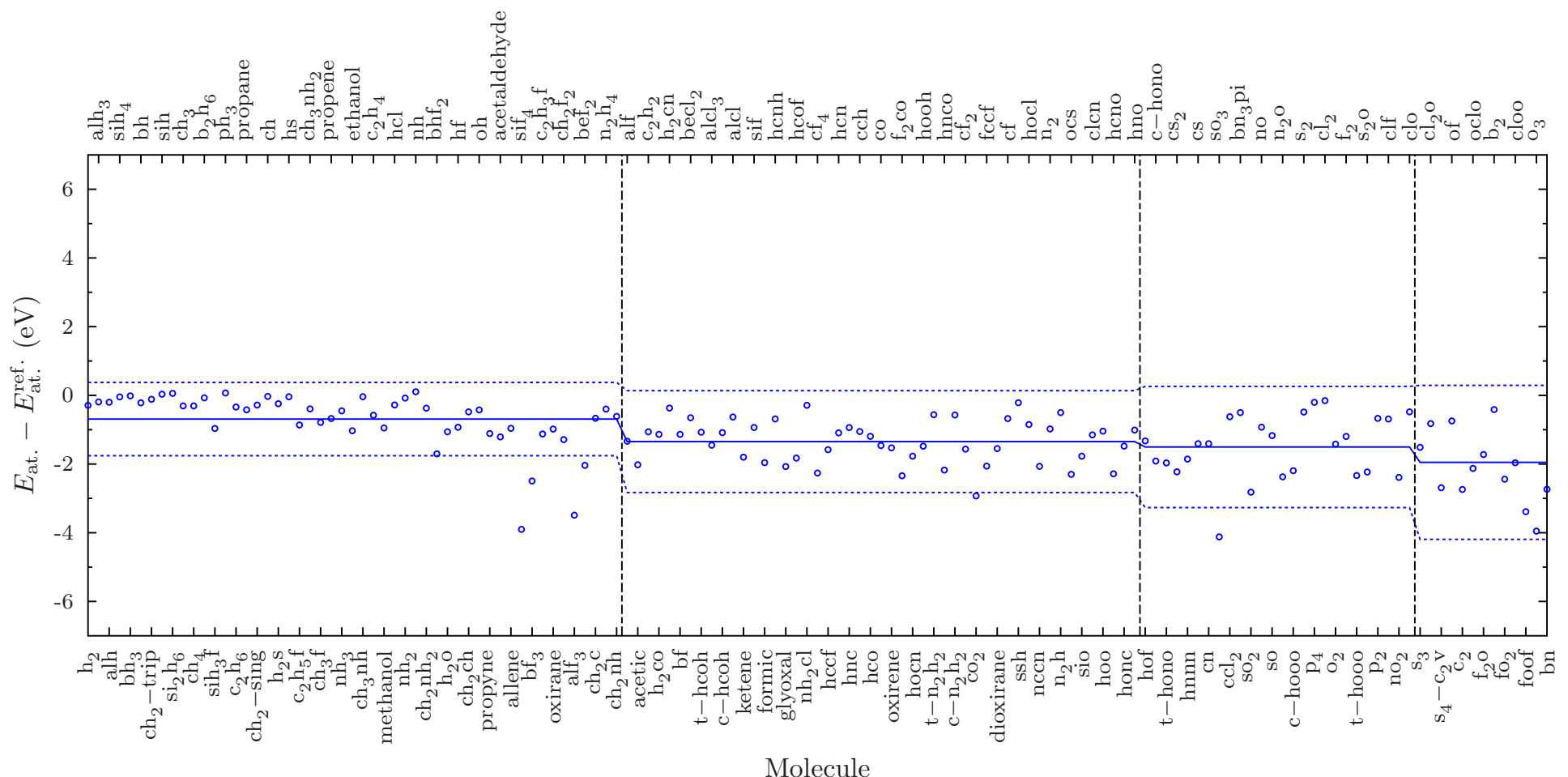

Figure 20: PBE0 PZ-RSIC results. 


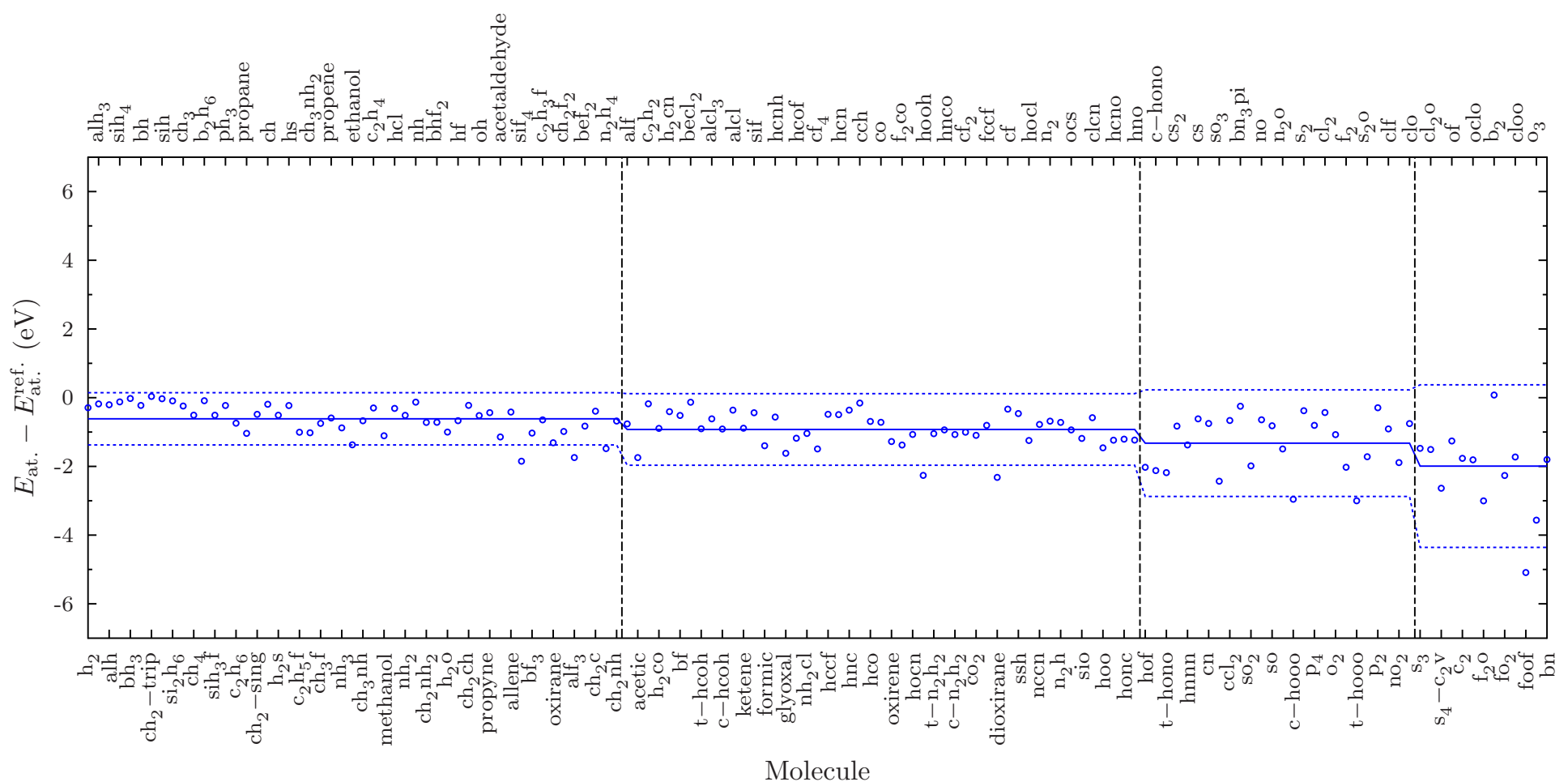

Figure 21: PBE0 PZ-SIC results.

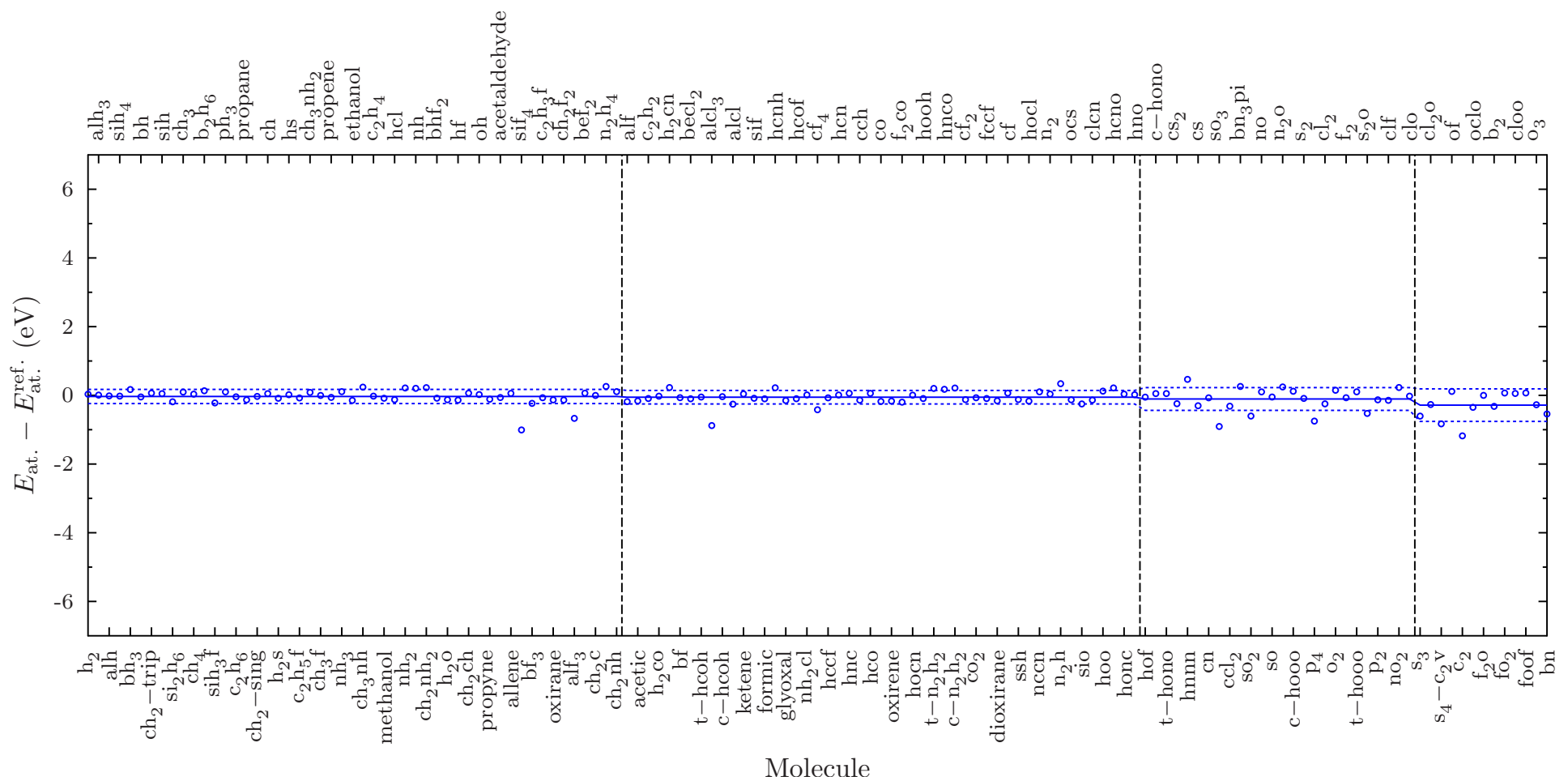

Figure 22: B3LYP KS-DFT results. 


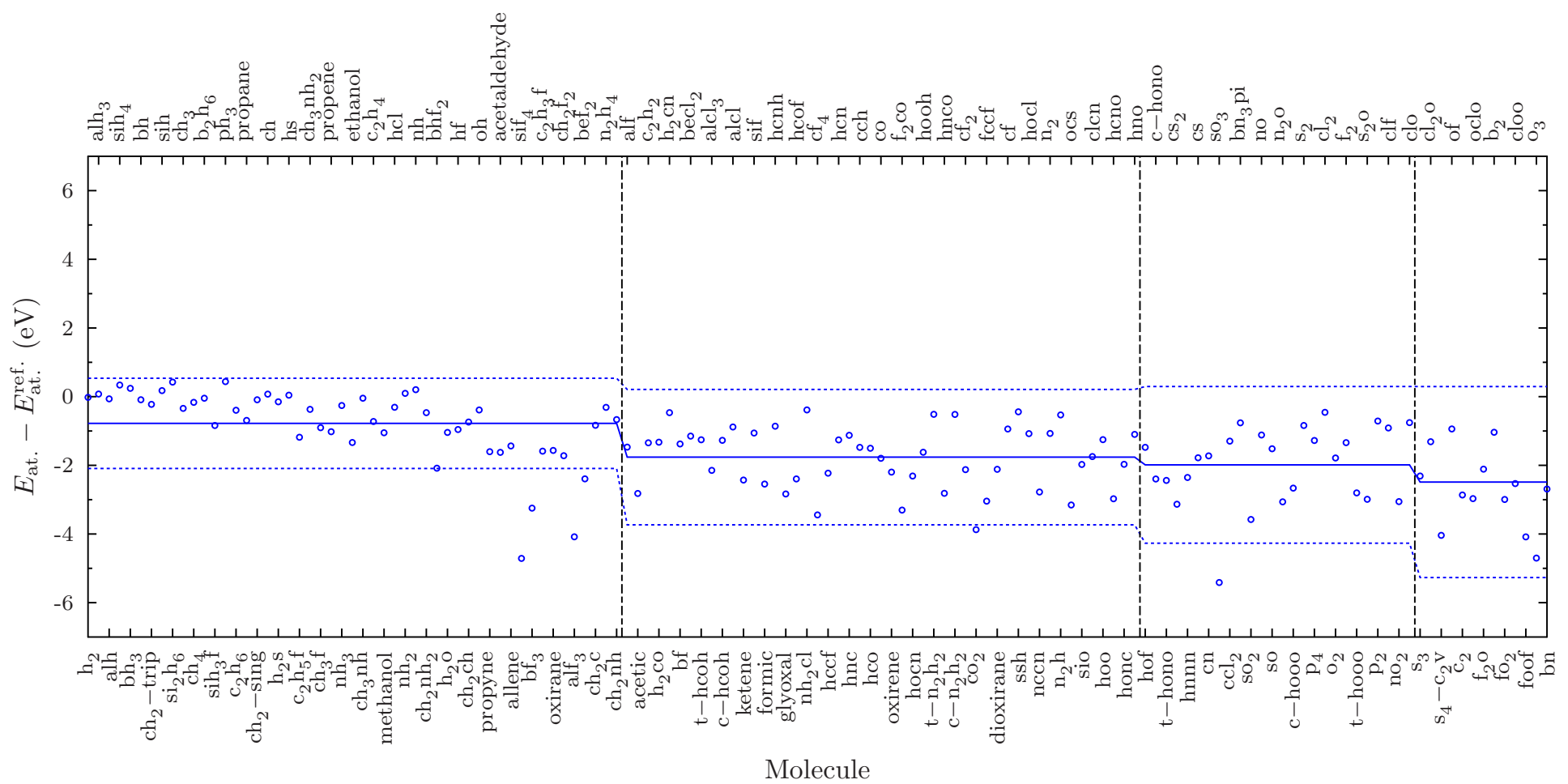

Figure 23: B3LYP PZ-RSIC results.

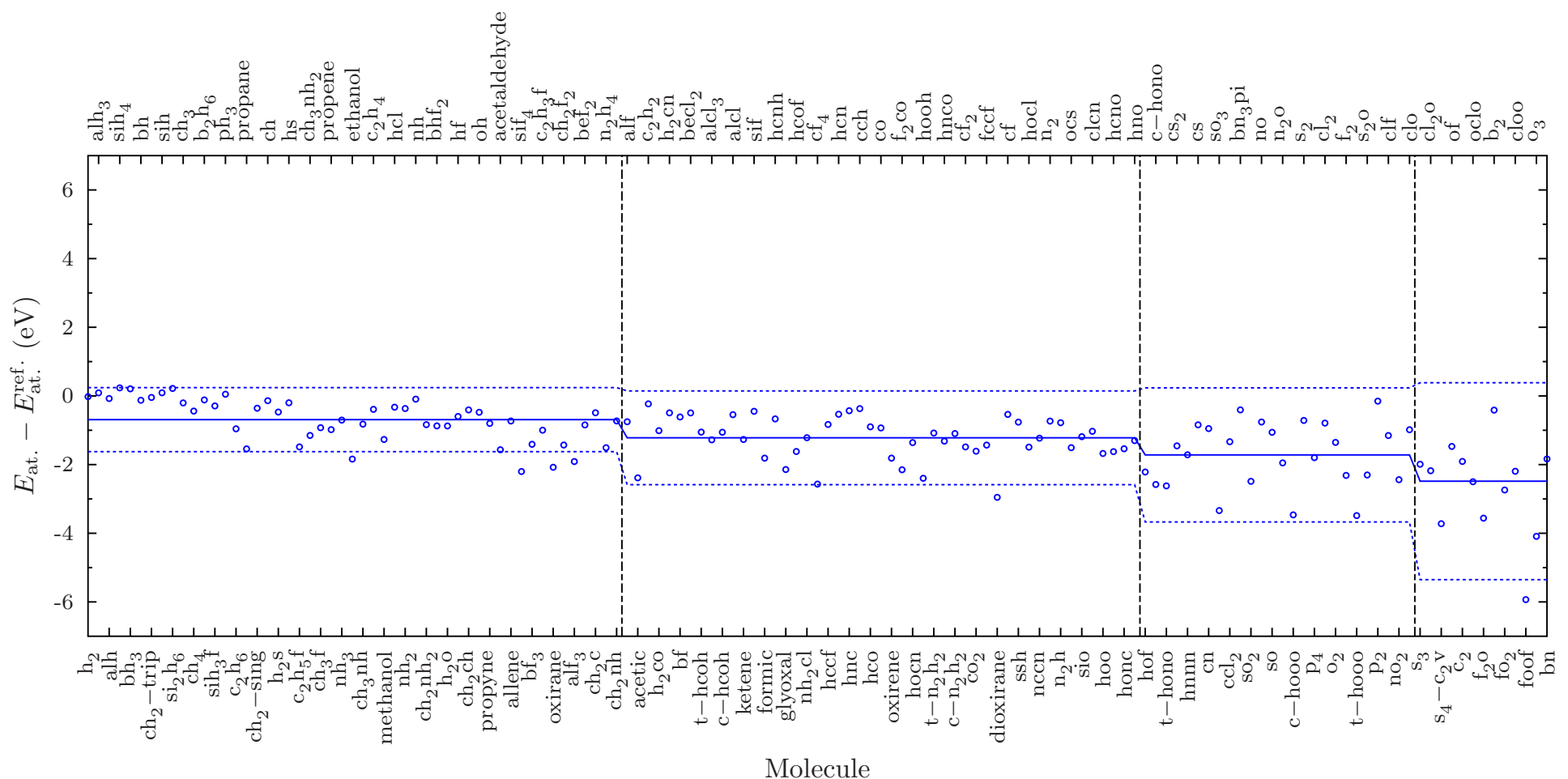

Figure 24: B3LYP PZ-SIC results. 


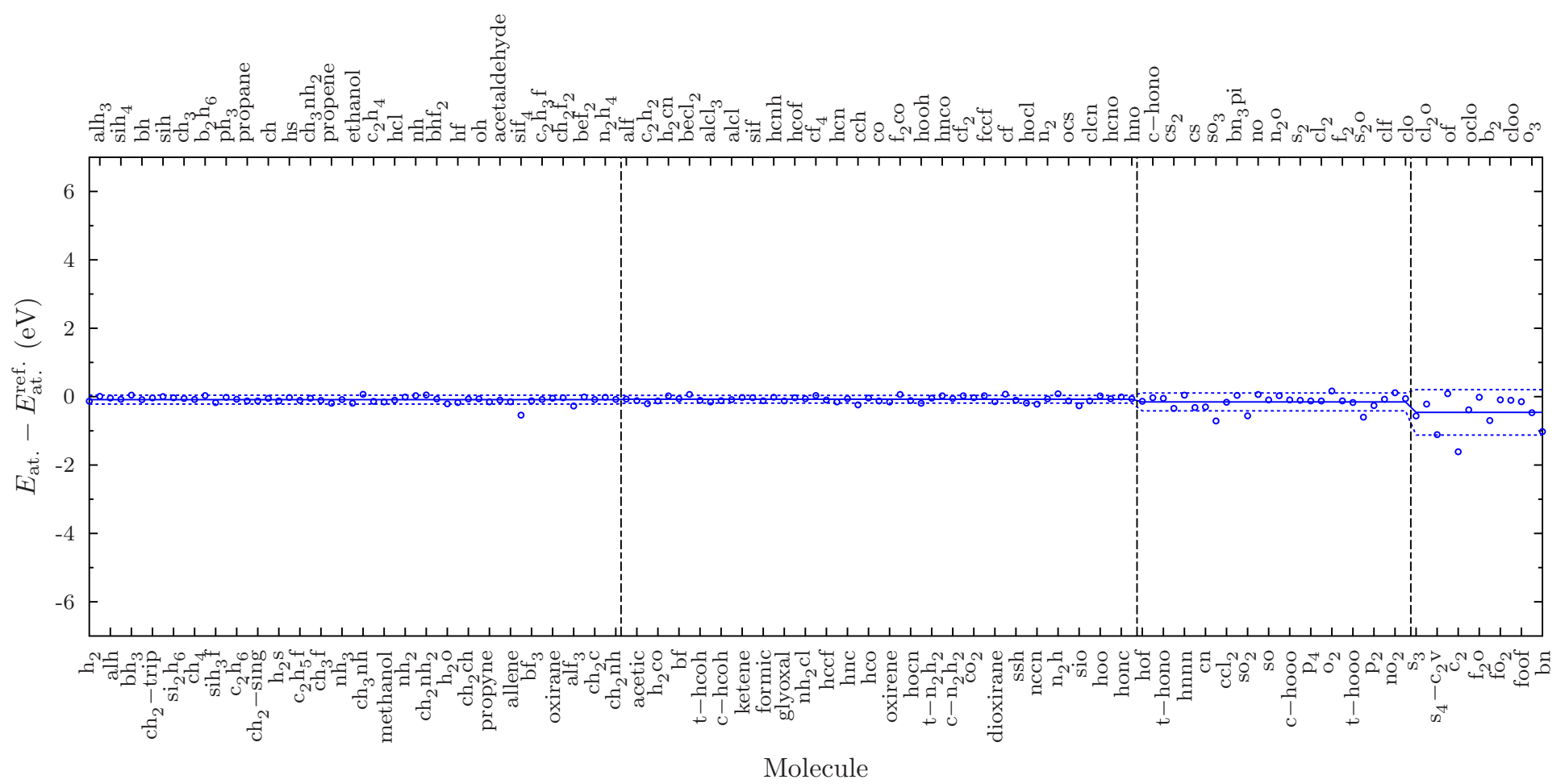

Figure 25: $\omega$ B97X KS-DFT results.

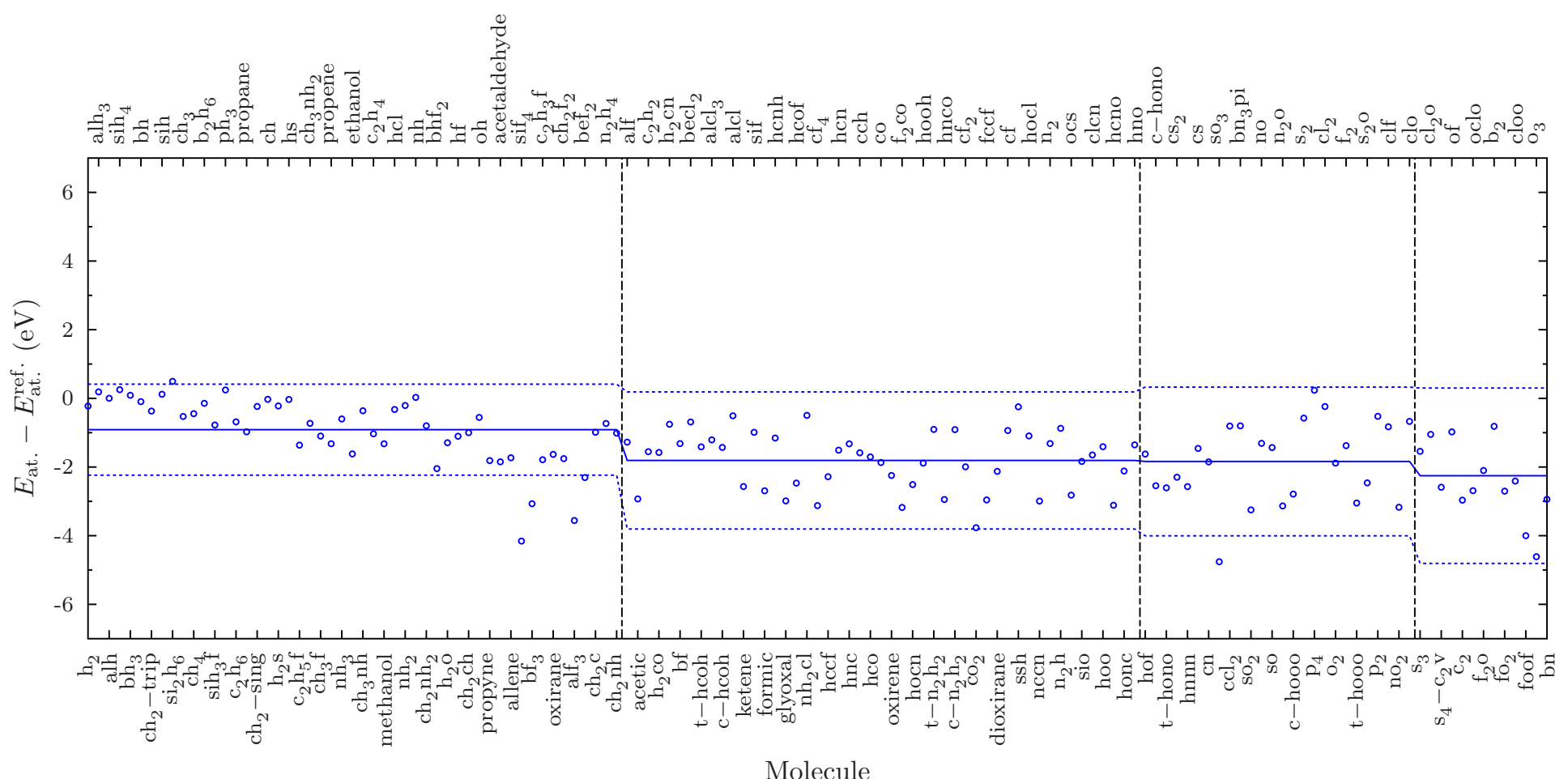

Figure 26: $\omega$ B97X PZ-RSIC results. 


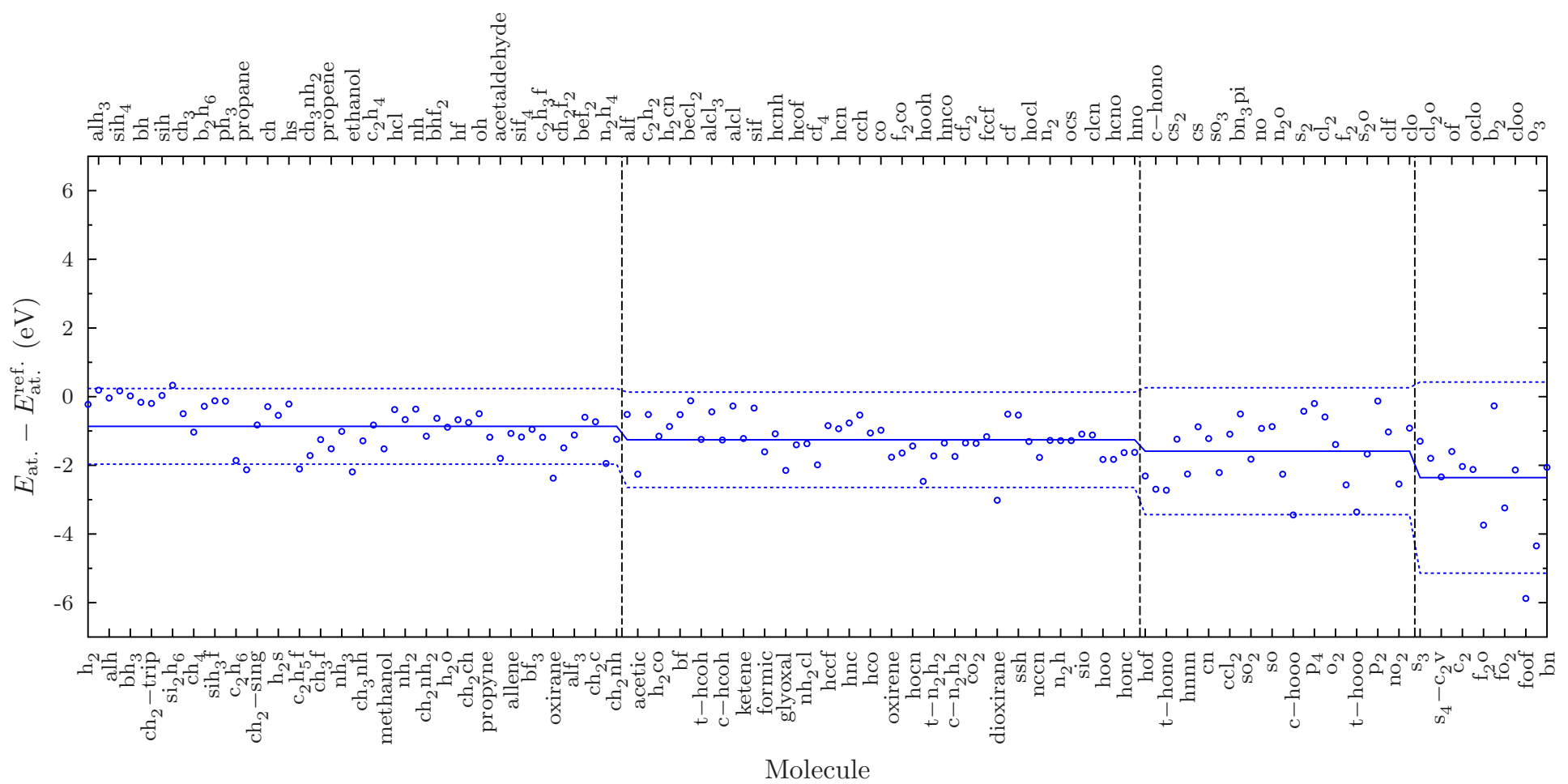

Figure 27: $\omega \mathrm{B} 97 \mathrm{X}$ PZ-SIC results.

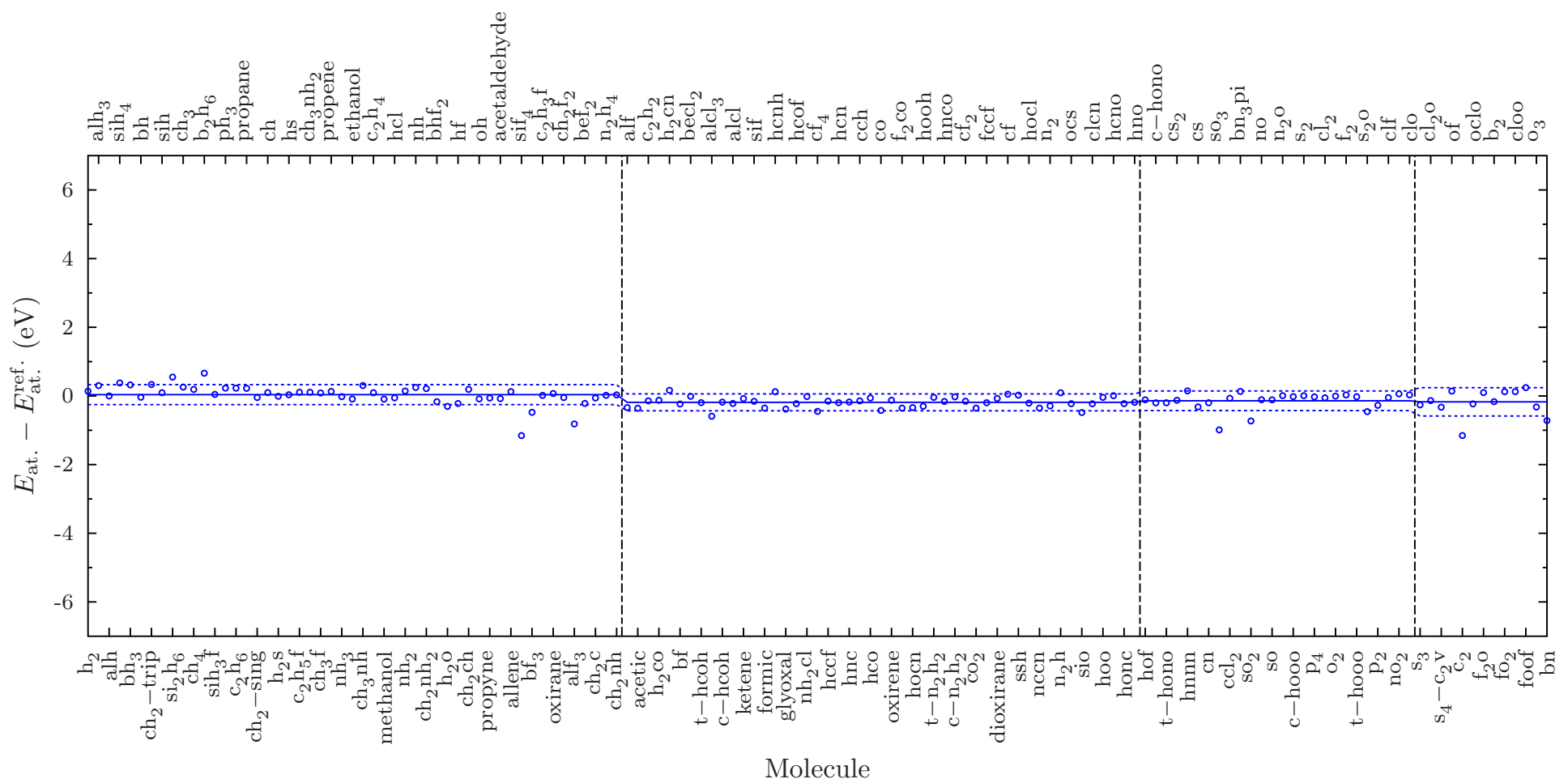

Figure 28: TPSSh KS-DFT results. 


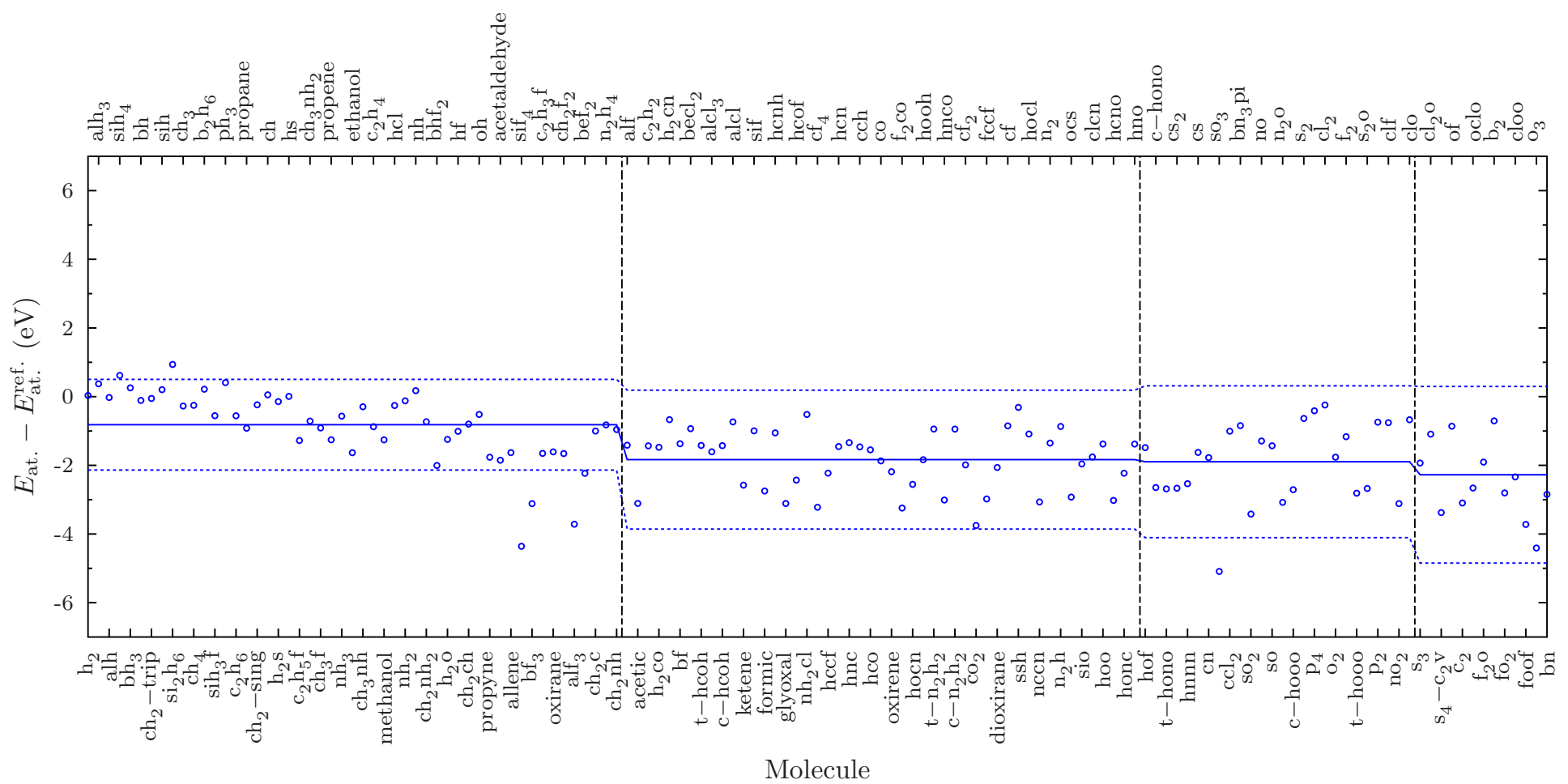

Figure 29: TPSSh PZ-RSIC results.

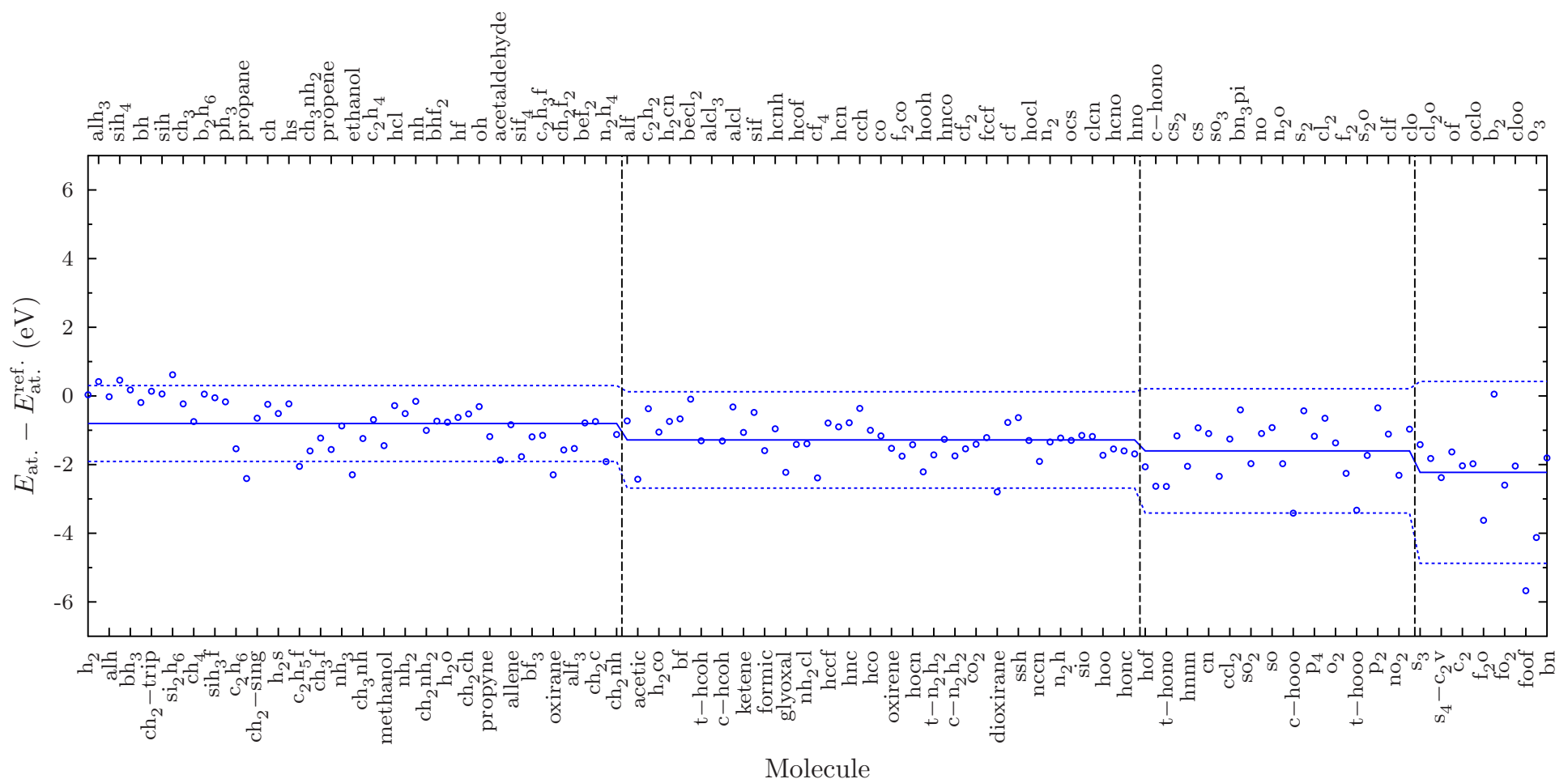

Figure 30: TPSSh PZ-SIC results. 


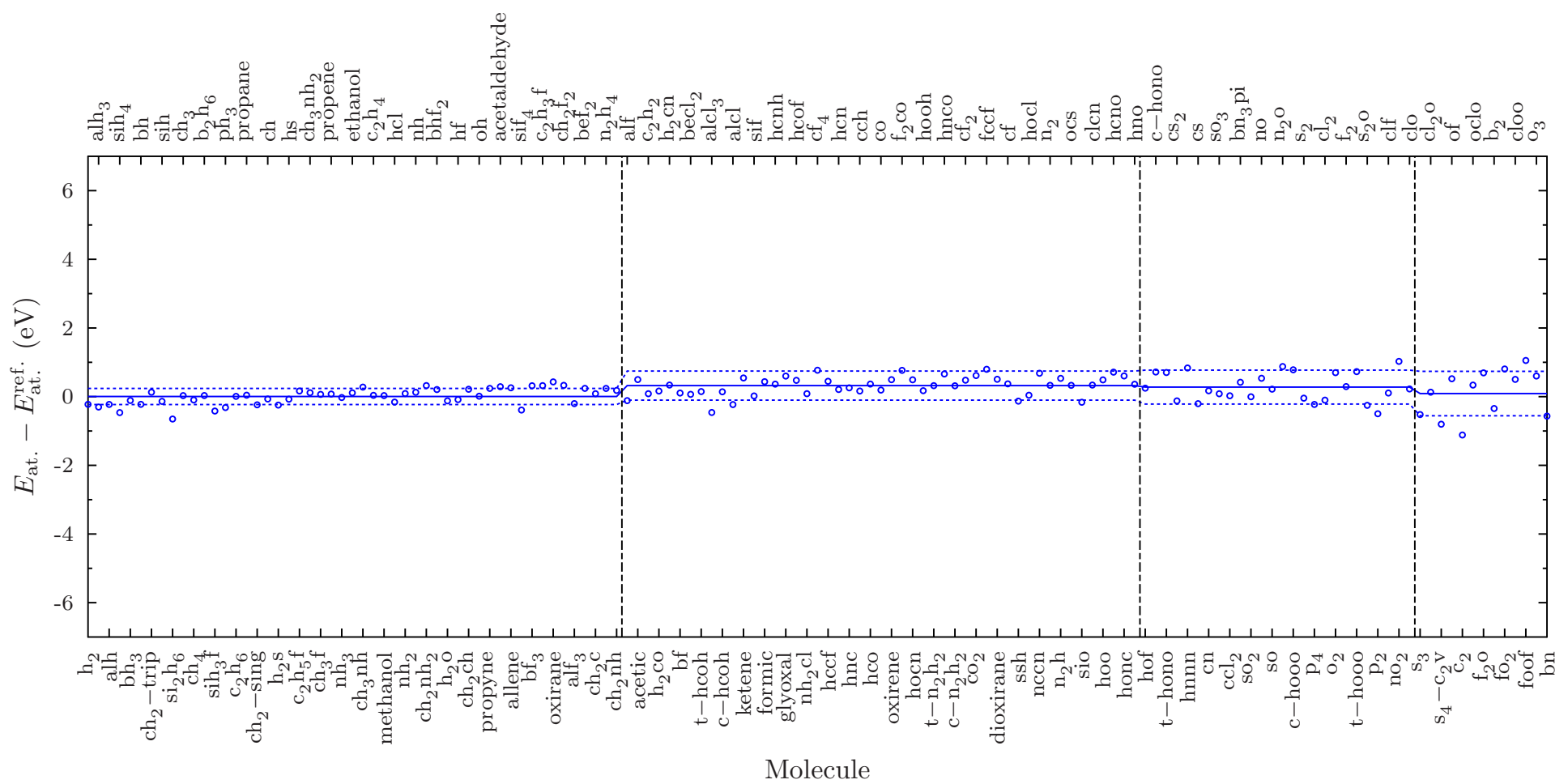

Figure 31: LRC- $\omega$ PBE KS-DFT results.

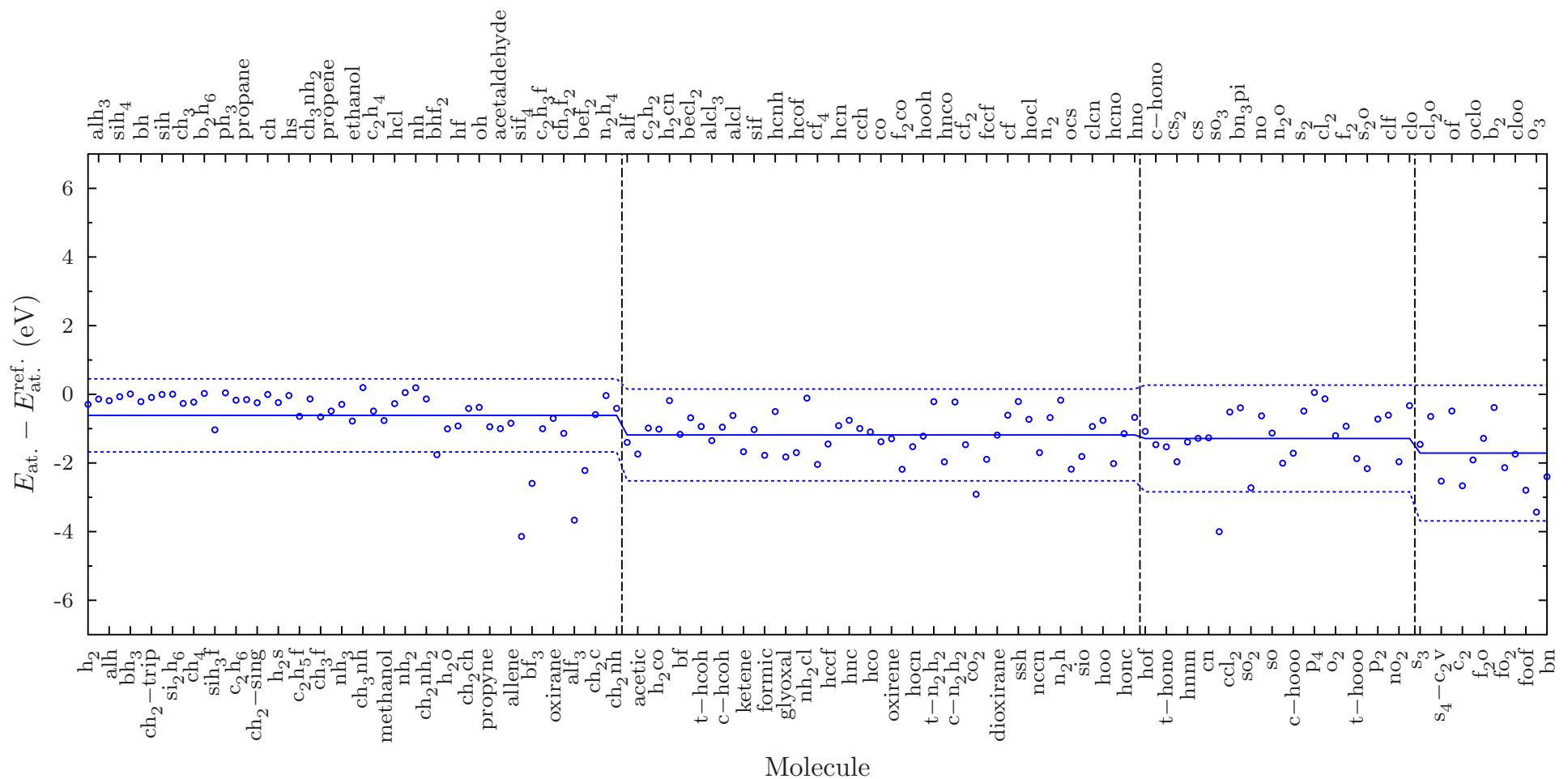

Figure 32: LRC- $\omega$ PBE PZ-RSIC results. 


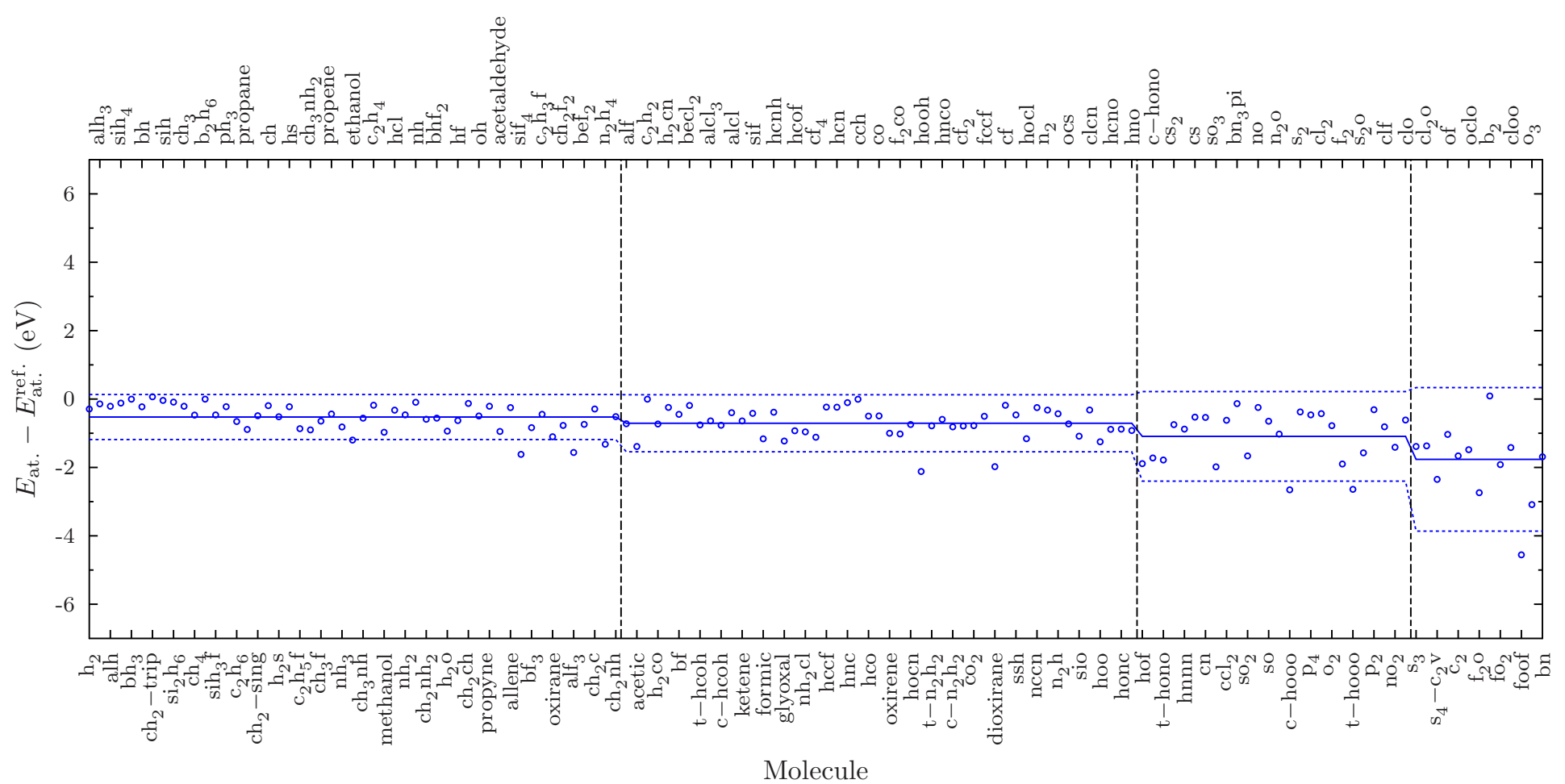

Figure 33: LRC- $\omega$ PBE PZ-SIC results.

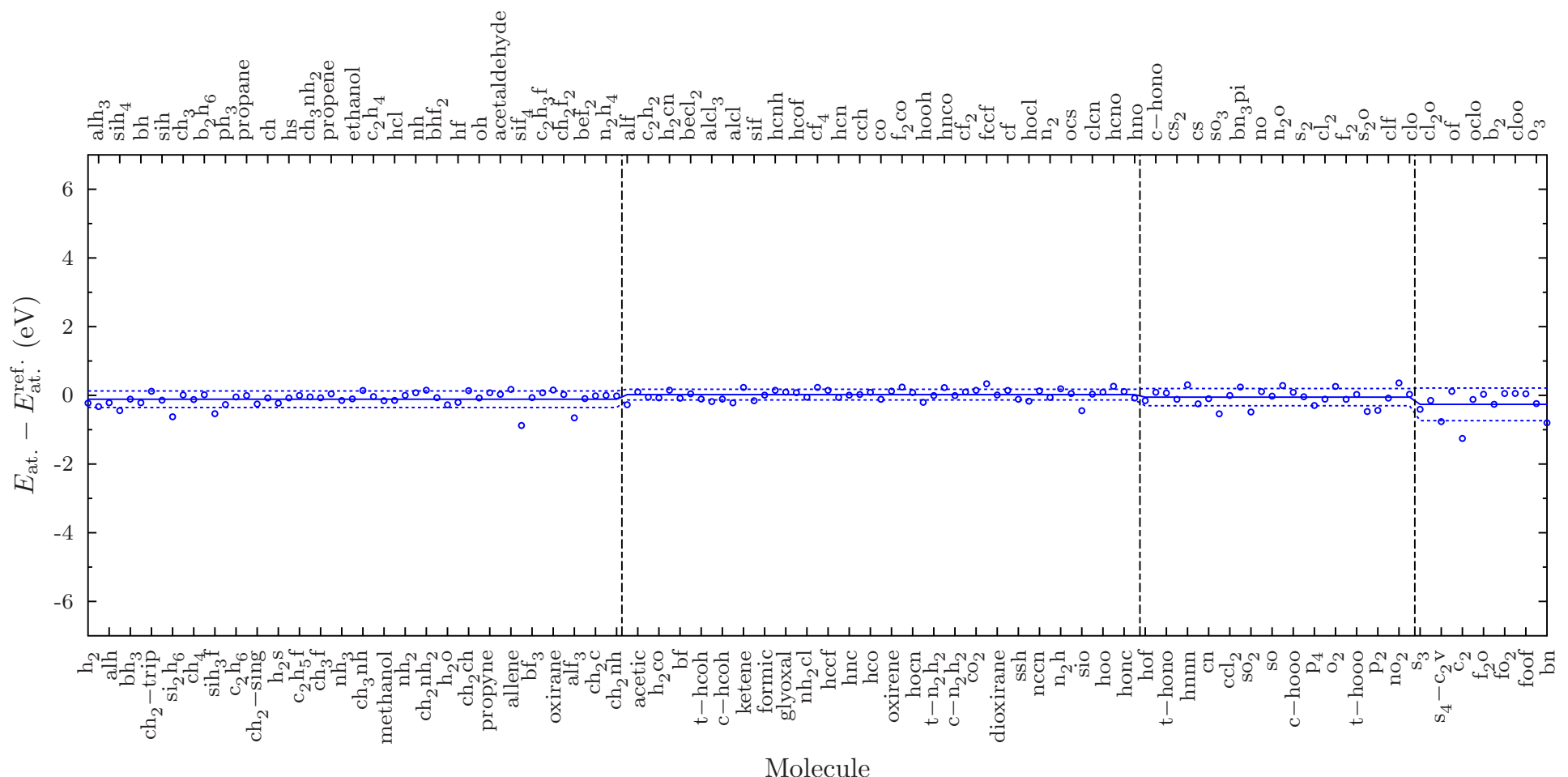

Figure 34: LRC- $\omega$ PBEh KS-DFT results. 


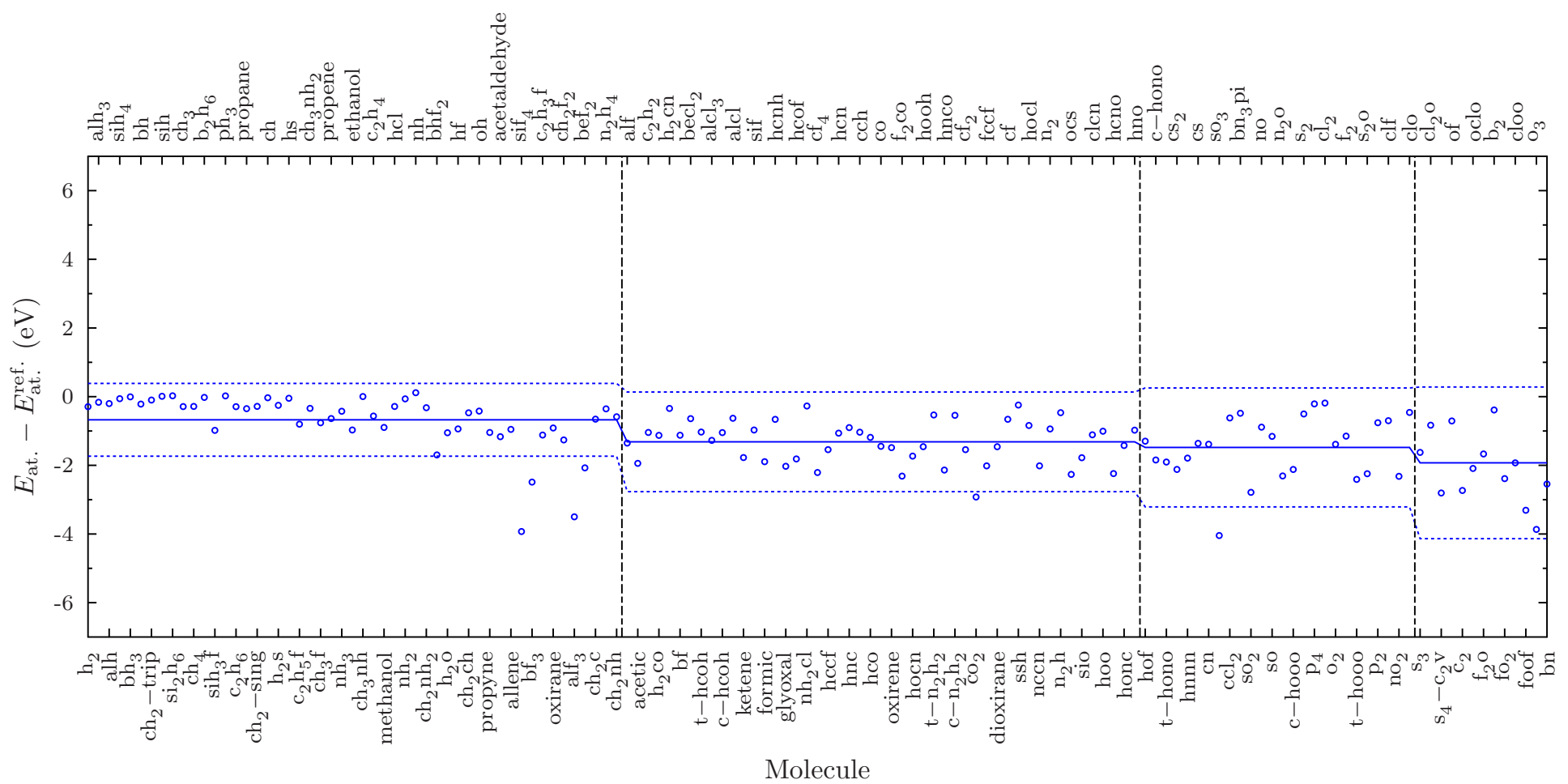

Figure 35: LRC- $\omega$ PBEh PZ-RSIC results.

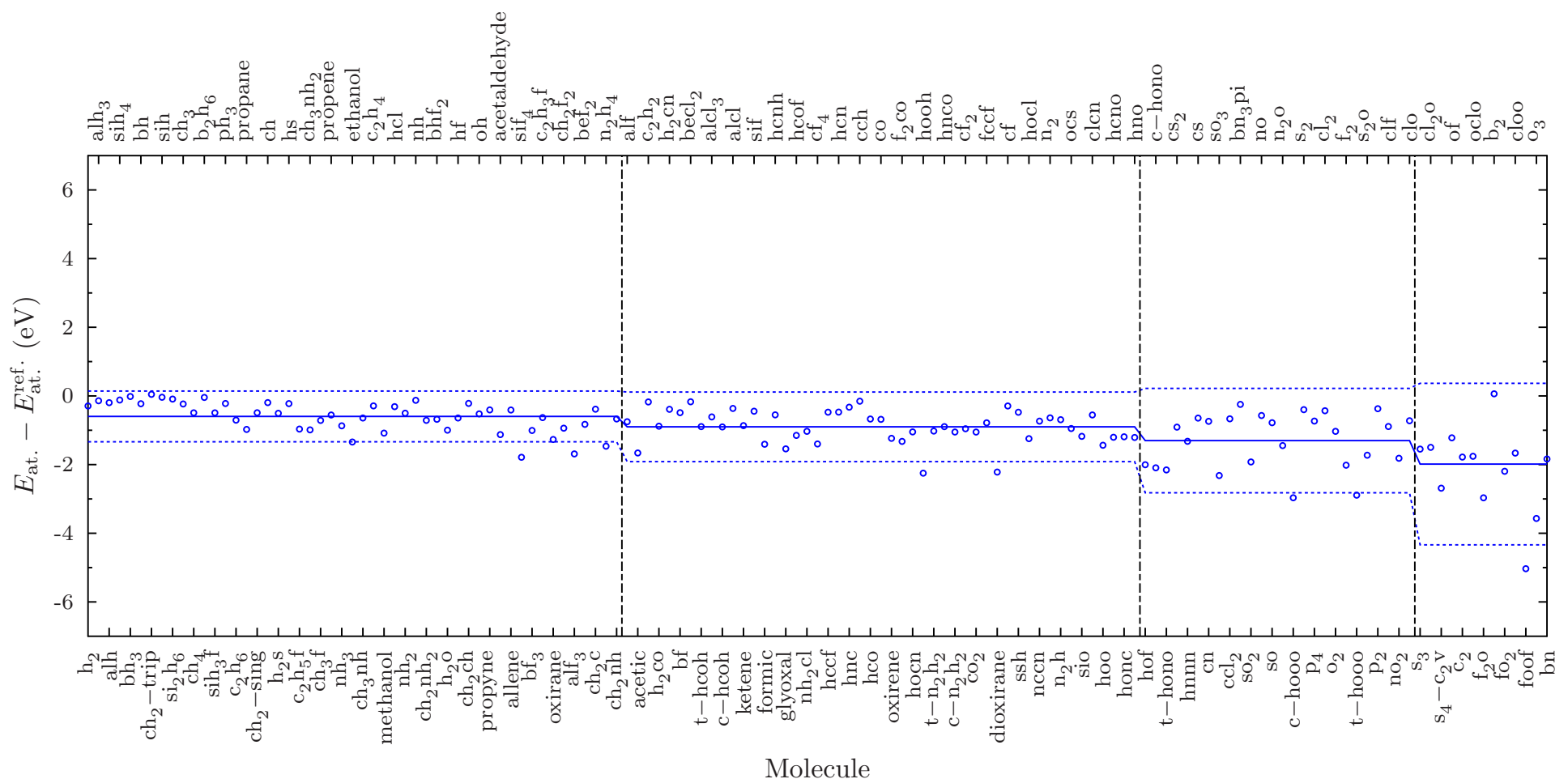

Figure 36: LRC- $\omega$ PBEh PZ-SIC results. 